\title{
A new perspective on the integrability of Inozemtsev's elliptic spin chain
}

\author{
Federico Finkel, Artemio González-López* \\ Departamento de Física Teórica II, Universidad Complutense de Madrid, 28040 Madrid, Spain
}

\begin{abstract}
The aim of this paper is studying from an alternative point of view the integrability of the spin chain with long-range elliptic interactions introduced by Inozemtsev. Our analysis relies on some well-established conjectures characterizing the chaotic vs. integrable behavior of a quantum system, formulated in terms of statistical properties of its spectrum. More precisely, we study the distribution of consecutive levels of the (unfolded) spectrum, the power spectrum of the spectral fluctuations, the average degeneracy, and the equivalence to a classical vertex model. Our results are consistent with the general consensus that this model is integrable, and that it is closer in this respect to the Heisenberg chain than to its trigonometric limit (the Haldane-Shastry chain). On the other hand, we present some numerical and analytical evidence showing that the level density of Inozemtsev's chain is asymptotically Gaussian as the number of spins tends to infinity, as is the case with the Haldane-Shastry chain. We are also able to compute analytically the mean and the standard deviation of the spectrum, showing that their asymptotic behavior coincides with that of the Haldane-Shastry chain.
\end{abstract}

Keywords: Spin chains with long-range interactions, Integrability vs. quantum chaos

\section{Introduction}

The celebrated spin chain with long-range interactions introduced independently by Haldane [1] and Shastry [2] in 1988 as a toy model for the one-dimensional Hubbard Hamiltonian has attracted considerable interest in Mathematical Physics, due to its remarkable integrability and solvability properties. To name only a few, the $\operatorname{su}(m)$ Haldane-Shastry (HS) chain is completely integrable [3], it is invariant under the Yangian $Y(\operatorname{su}(m))$ [4], its partition function can be exactly evaluated for an arbitrary number of spins [5], and it is equivalent to a simple classical vertex model [6]. As a matter of fact, the latter properties can be ultimately traced back to the connection of this chain to the dynamical spin Sutherland (trigonometric) model [7] via the socalled "freezing trick" of Polychronakos [8]. Roughly speaking, in the strong coupling limit the dynamical degrees of freedom of the latter model decouple from the spin ones, which are in turn governed by the Hamiltonian of the HS chain. Thus, for instance, the chain's spectrum can be

*Corresponding author

Email address: artemio@ucm.es (Artemio González-López) 
obtained essentially by "modding out" the energies of the scalar Sutherland model [9, 10] from the spectrum of its spin counterpart.

The fact that the HS chain can be obtained in a suitable limit from a dynamical spin model is perhaps the feature that sets it apart from other integrable models like, e.g., the Heisenberg chain. This idea has also been successfully applied to the Calogero (rational) [11, 12] and Inozemtsev (hyperbolic) [13, 14] integrable spin dynamical models, which respectively yield the Polychronakos-Frahm [8, 15] and Frahm-Inozemtsev [16] chains. The Haldane-Shastry, Polychronakos-Frahm and Frahm-Inozemtsev chains (which shall be collectively referred to as spin chains of HS type), and their corresponding dynamical models, are all associated with the $A_{N-1}$ root system [17], where $N$ is the number of spins. As is well known, there are generalizations of these models and of their related spin chains to all the other (extended) root systems (see, e.g., [17]), although we shall not deal with these more general models in this paper.

The rational, trigonometric and hyperbolic models of Calogero-Sutherland (CS) type associated with the $A_{N-1}$ root system are all limiting cases of a more general model with a two-body interaction potential proportional to a Weierstrass $\wp$ function with suitable periods [17]. This elliptic CS model, which can be regarded as the $N$-dimensional version of the Lamé potential, is also completely integrable [17]. On the other hand, in spite of extensive work by many authors (see, e.g., [18-[22]), there are no general explicit formulas for the eigenvalues and eigenfunctions of this model. Even less is known about its spin version, for which only the lowest integrals of motion [23] and a few particular solutions (in the three-particle case) have been found [24]. Notwithstanding this, it is natural to consider the spin chain obtained by applying Polychronakos's freezing trick to the elliptic CS model. Remarkably, as we shall prove in this paper, this is essentially the chain introduced by Inozemtsev in 1990 as a model encompassing both the Haldane-Shastry and Heisenberg chains [25]. It should be pointed out that in Inozemtsev's original construction, the functional form of the spin-spin interaction is obtained not through the freezing trick (which was actually formulated a few years later), but by requiring that the Hamiltonian possess a quantum Lax pair analogous to that of the classical elliptic CS model.

The interest in Inozemtsev's elliptic chain has been recently rekindled due to its relevance in connection with the AdS-CFT correspondence. Indeed, this long-range spin chain has been advanced as a candidate for describing planar $\mathcal{N}=4$ gauge theory non-perturbatively [26-29]. Since the latter theory is believed to be integrable in view of the AdS-CFT correspondence, an essential requirement for the validity of this description is the integrability of the Inozemtsev chain. In fact, already in Inozemtsev's original paper two first integrals in involution were explicitly exhibited. In a subsequent work [30], the same author constructed a large family of first integrals including the two previously known ones. Although it is conjectured that this family contains a maximal set of first integrals in involution [31], to the best of our knowledge this crucial fact has never been rigorously established. Thus, technically speaking the integrability of Inozemtsev's chain still remains an open question 1 , which has defied the efforts of many specialists in the field for over two decades.

The main aim of this paper is the analysis of the integrability of Inozemtsev's chain from a different perspective, based on the statistical properties of its spectrum in the framework of several important conjectures in quantum chaos. The first of these conjectures, formulated by Berry and Tabor in the late seventies [32], posits that the distribution of the spacings between

\footnotetext{
${ }^{1}$ It is true that, as remarked above, Inozemtsev's chain admits a quantum Lax pair by construction. It should be noted, however, that a quantum Lax pair by no means guarantees the existence of first integrals; see, e.g., [25]. Indeed, in the quantum case the entries of the Lax matrices are operators, which in general do not commute with each other.
} 
consecutive (suitably normalized) levels of a "generic" integrable quantum system should be Poissonian. By contrast, in the case of a fully chaotic quantum system the spacings distribution should follow a Wigner-type law (cf., for instance, Eq. (4.1) below). In fact, the latter conjecture, due to Bohigas, Giannoni and Schmit [33], is one of the cornerstones of the theory of quantum chaos. In view of these conjectures, the behavior of the spacings distribution of a quantum system can be used as a basic integrability test, relying exclusively on statistical properties of its spectrum. It is well known, for instance, that the Heisenberg (spin 1/2) chain passes this integrability test [34]. In this paper we will show that this is also the case for Inozemtsev's chain, which provides further evidence of its integrability.

An alternative statistical test of integrability, based on treating the energy levels as a time sequence, has been recently proposed in Refs. [35, 36]. According to this test, the power spectrum $\mathcal{P}(v)$ of the spectral fluctuations of an integrable quantum system should behave as $v^{-2}$ for sufficiently small values of the frequency $v$, while in a chaotic system $\mathcal{P}(v) \sim v^{-1}$. We shall see that the power spectrum of Inozemtsev's chain follows the first of these power laws, hinting again at its integrability. For comparison purposes, we shall also study the power spectrum of Heisenberg's chain, showing that it also behaves as the inverse square of the frequency, as expected for an integrable model.

The invariance of the Haldane-Shastry and Polychronakos-Frahm chains under the full Yangian algebra implies that their spectrum is highly degenerate. Another important consequence of the Yangian symmetry is the equivalence of the latter chains to a simple classical vertex model (cf. Eq. (4.3) below), whose interactions are closely related to Haldane's motifs [4]. On the other hand, it is known that the Heisenberg chain is only invariant under the Yangian in the limit when the number of spins tends to infinity [37], which also explains why its spectrum is much less degenerate. It is widely believed that, like the Heisenberg chain, Inozemtsev's chain does not possess Yangian symmetry for a finite number of spins. In this work we shall devise a simple test to rule out the equivalence of a quantum system to a classical vertex model of the form (4.3). Applying this test we shall rigorously show that the spin $1 / 2$ Inozemtsev chain is not equivalent to a vertex model of the form (4.3) for $10 \leqslant N \leqslant 18$ and a wide range of values of the elliptic modulus. This strongly suggests that this is true in general, which is a further indication of the lack of Yangian symmetry of the model. Moreover, we shall also see that for $N \gtrsim 10$ the average degeneracy of Inozemtsev's chain is essentially equal to that of the Heisenberg chain. This is again consistent with the general consensus that Inozemtsev's chain is an integrable system, more akin to the Heisenberg than to the original Haldane-Shastry chain (which is obtained by setting the elliptic modulus to zero).

In spite of the latter conclusion, we shall see that Inozemtsev's elliptic chain shares several important properties with the HS chain. More precisely, it has been rigorously established [38] that the level density of all spin chains of HS type becomes normally distributed in the large $N$ limit. The parameters of this normal distribution coincide with the mean and the standard deviation of the spectrum, which have been computed in closed form [5] and are respectively $O\left(N^{3}\right)$ and $O\left(N^{5 / 2}\right)$. By numerically diagonalizing the Hamiltonian, we shall provide strong evidence that the level density of Inozemtsev's chain is also asymptotically Gaussian for sufficiently large $N$. To further support this conclusion, we shall rigorously show that the skewness and the excess kurtosis of the spectrum tend to zero as $N \rightarrow \infty$. We shall also compute in closed form both the mean and the standard deviation of the spectrum as a function of $N, m$ and the elliptic modulus, and show that they have the same asymptotic behavior when $N \rightarrow \infty$ as for the HS chain. To this end, we shall evaluate several sums involving positive powers of the Weierstrass elliptic function, which are of interest per se as a natural generalization of the corresponding classical formulas 
for the cosecant or cotangent [39].

The paper is organized as follows. In Section 2 we construct the spin chain associated with the elliptic spin Calogero-Sutherland model by means of Polychronakos's freezing trick, and explicitly show that it essentially coincides with Inozemtsev's elliptic chain. Section 3 is devoted to the study of the level density of Inozemtsev's chain, and to the evaluation of the mean and variance of its spectrum in closed form. In Section 4 we present our statistical analysis of the chain's integrability, studying the distribution of the spacings between consecutive levels, the power spectrum of the spectral fluctuations, and the equivalence to a classical vertex model. The paper ends with four appendices covering some background material on elliptic functions, as well as the proofs of some technical results used in the body of the paper. In particular, in Appendix C we show how to evaluate in closed form certain finite sums of powers of the Weierstrass elliptic function, and determine their leading asymptotic behavior.

\section{The spin chain}

Apart from an irrelevant overall factor, the Hamiltonian of the scalar Calogero-Sutherland model (of $A$ type) with elliptic interactions is commonly taken as

$$
H^{\mathrm{sc}}=-\Delta+a(a-1) \sum_{1 \leqslant i \neq j \leqslant N} \wp\left(x_{i}-x_{j}\right) .
$$

Note that the half-periods $\omega_{1}, \omega_{3}$ of the Weierstrass elliptic function ${ }^{2} \wp$ are assumed to satisfy the condition $\omega_{1}, \mathrm{i} \omega_{3} \in \mathbb{R}$, so that $\wp\left(x_{i}-x_{j}\right)$ is real and, consequently, $H^{\text {sc }}$ is self-adjoint in the Weyl alcove

$$
x_{1}<\cdots<x_{N}<x_{1}+2 \omega_{1} .
$$

The $\operatorname{su}(m)$ spin version of the previous model is given by

$$
H=-\Delta+\sum_{1 \leqslant i \neq j \leqslant N} \wp\left(x_{i}-x_{j}\right) a\left(a-S_{i j}\right),
$$

where $S_{i j}$ is the operator permuting the $i$-th and $j$-th spins. More precisely, the action of $S_{i j}$ on an element $|\mathbf{s}\rangle \equiv\left|s_{1}, \ldots, s_{N}\right\rangle \equiv\left|s_{1}\right\rangle \otimes \cdots \otimes\left|s_{N}\right\rangle, 1 \leqslant s_{i} \leqslant m$, of the canonical basis of the internal Hilbert space $\Sigma \equiv\left(\mathbb{C}^{m}\right)^{\otimes N}$ is given by

$$
S_{i j}\left|s_{1}, \ldots, s_{i}, \ldots, s_{j}, \ldots, s_{N}\right\rangle=\left|s_{1}, \ldots, s_{j}, \ldots, s_{i}, \ldots, s_{N}\right\rangle .
$$

Using Eqs. A.7, A.12 and A.13, and performing the change of variables $x_{i}^{\prime}=\sqrt{e_{1}-e_{3}} x_{i}$, we immediately obtain

$$
H=\left(e_{1}-e_{3}\right)\left(-\Delta^{\prime}+\sum_{1 \leqslant i \neq j \leqslant N} \frac{a\left(a-S_{i j}\right)}{\operatorname{sn}^{2}\left(x_{i}^{\prime}-x_{j}^{\prime}\right)}\right)+e_{3} \sum_{1 \leqslant i \neq j \leqslant N} a\left(a-S_{i j}\right) .
$$

The constant operator $\sum_{1 \leqslant i \neq j \neq N} S_{i j}$ commutes with $x_{i}, \partial_{x_{i}}$ and $S_{i j}$ for all $i, j$, and hence with $H$. Therefore the last term in $H$ can be omitted without changing neither the integrability nor the

\footnotetext{
${ }^{2}$ We refer the reader to Appendix A for a brief summary of several basic properties of the Weierstrass and Jacobi elliptic functions needed in the sequel.
} 
solvability of the model. We shall thus equivalently define the Hamiltonian of the elliptic spin Calogero-Sutherland model as

$$
H=-\Delta+\sum_{1 \leqslant i \neq j \leqslant N} \frac{a\left(a-S_{i j}\right)}{\mathrm{sn}^{2}\left(x_{i}-x_{j}\right)} .
$$

This definition has several practical advantages over the usual one. In particular, the operator $H$ in the latter equation depends on a single real parameter $k \in[0,1)$ (the modulus of the elliptic function), the value $k=0$ yielding the well-known expression for the spin Sutherland model. The scalar counterpart of Eq. (2.3) is

$$
H^{\mathrm{sc}}=-\Delta+\sum_{1 \leqslant i \neq j \leqslant N} \frac{a(a-1)}{\operatorname{sn}^{2}\left(x_{i}-x_{j}\right)},
$$

which differs from the Hamiltonian 2.1p by a trivial (constant) rescaling and the addition of a constant energy. Note that the configuration space of both $H$ and $H^{\mathrm{sc}}$ can be taken as the Weyl alcove

$$
A=\left\{\mathbf{x} \in \mathbb{R}^{N}: x_{1}<\cdots<x_{N}<x_{1}+2 K\right\},
$$

where $2 K$ is the real period of the elliptic function $\mathrm{sn}^{2}$ (cf. Eq. A.9p).

From Eqs. 2.3 -2.4 it immediately follows that

$$
H=H^{\mathrm{sc}}+4 a \hat{H}(\mathbf{x}),
$$

with

$$
\hat{H}(\mathbf{x})=\frac{1}{2} \sum_{1 \leqslant i<j \leqslant N} \frac{1-S_{i j}}{\operatorname{sn}^{2}\left(x_{i}-x_{j}\right)} .
$$

In order to define the spin chain associated with the spin dynamical model [2.3), we need to study the equilibria in the Weyl alcove (2.5) of the function

$$
U(\mathbf{x})=\sum_{1 \leqslant i \neq j \leqslant N} \frac{1}{\operatorname{sn}^{2}\left(x_{i}-x_{j}\right)},
$$

which is proportional to the interaction potential of the scalar model (2.4). We shall show in Subsection 2.1 that (up to a rigid translation) $U$ has a unique minimum $\boldsymbol{\xi} \equiv\left(\xi_{1}, \ldots, \xi_{N}\right)$ in the set $A$, whose coordinates are given by

$$
\xi_{j}=\frac{2 j K}{N}, \quad j=1, \ldots, N .
$$

According to Polychronakos's freezing trick argument [8], the Hamiltonian of the spin chain associated with $H$ is (proportional to)

$$
\mathcal{H}=\hat{H}(\xi) \equiv \frac{1}{2} \sum_{1 \leqslant i<j \leqslant N} \frac{1-S_{i j}}{\operatorname{sn}^{2}\left(2(i-j) \frac{K}{N}\right)} .
$$

Moreover, it can be shown [40] that the partition functions $\mathcal{Z}, Z$ and $Z^{\text {sc }}$ of $\mathcal{H}, H$ and $H^{\text {sc }}$ are related by

$$
\mathcal{Z}(T)=\lim _{a \rightarrow \infty} \frac{Z(4 a T)}{Z^{\mathrm{sc}}(4 a T)} .
$$


In order to establish the equivalence between Eq. (2.8) and the original definition of Inozemtsev [25], it suffices to note that by Eqs. [A.1] and (A.11] we have

$$
\operatorname{sn}^{-2}\left(2(i-j) \frac{K}{N}\right)=\wp\left(2(i-j) \frac{K}{N} ; K, \mathrm{i} K^{\prime}\right)+\frac{1}{3}\left(1+k^{2}\right)=\frac{N^{2}}{4 K^{2}} \wp_{N}(i-j)+\frac{1}{3}\left(1+k^{2}\right),
$$

where $\wp_{N}$ is the Weierstrass function with periods $N$ and $\mathrm{i} N K^{\prime} / K \equiv \mathrm{i} N \tau$.

It is obvious on account of Eq. A.10) that when $k=0$ the elliptic chain (2.8) reduces exactly to the Haldane-Shastry chain

$$
\mathcal{H}_{\mathrm{HS}}=\frac{1}{2} \sum_{1 \leqslant i<j \leqslant N} \frac{1-S_{i j}}{\sin ^{2}\left((i-j) \frac{\pi}{N}\right)} .
$$

(This is, indeed, the main reason to modify Inozemtsev's original definition.) It is also of interest to study the limit of the Hamiltonian (2.8) when $k \rightarrow 1$. To this ends, we shall make use of the identity

$$
\frac{\omega_{1}^{2}}{\pi^{2}} \wp\left(z ; \omega_{1}, \omega_{3}\right)=-\frac{1}{12}+\frac{1}{4} \sin ^{-2}\left(\frac{\pi z}{2 \omega_{1}}\right)+4 \sum_{j=1}^{\infty} \frac{j q^{2 j}}{1-q^{2 j}} \sin ^{2}\left(\frac{j \pi z}{2 \omega_{1}}\right)
$$

(cf. [41], Eqs. 23.8.1-5), where $\operatorname{Im}\left(\omega_{3} / \omega_{1}\right)>0$ and

$$
q=\mathrm{e}^{\mathrm{i} \pi \omega_{3} / \omega_{1}}
$$

is the so-called Jacobi nome. From Eqs. 22.9-2.11] with $\omega_{1}=-\mathrm{i} K^{\prime}, \omega_{3}=K$ and $z=2 l K / N \equiv$ $2 l \omega_{3} / N$, after a straightforward calculation we obtain

$$
\operatorname{sn}^{-2}(2 l K / N)=\frac{1}{3}\left(1+k^{2}\right)+\frac{\pi^{2}}{K^{\prime 2}}\left(\frac{1}{12}+\frac{q^{2 l / N}}{\left(1-q^{2 l / N}\right)^{2}}+\sum_{j=1}^{\infty} \frac{j q^{2 j(N-l) / N}\left(1-q^{2 j l / N}\right)^{2}}{1-q^{2 j}}\right) .
$$

Letting $k \rightarrow 1$ and noting that $K^{\prime}(1)=\pi / 2$ and $q \rightarrow 0$ as $k \rightarrow 1$ we finally have

$$
\lim _{k \rightarrow 1} \operatorname{sn}^{-2}(2 l K / N)=1, \quad 1 \leqslant l \leqslant N-1,
$$

and therefore

$$
\lim _{k \rightarrow 1} \mathcal{H}=\frac{1}{2} \sum_{i<j}\left(1-S_{i j}\right) \equiv \mathcal{H}_{1}
$$

As first pointed out by Inozemtsev, the Heisenberg chain is also related to the $k \rightarrow 1$ limit of the elliptic chain. Indeed, when $k \rightarrow 1$ the nome $q$ and the imaginary half-period $\mathrm{i} K^{\prime}$ satisfy ${ }^{3}$

$$
q=\frac{\varepsilon}{16}+O\left(\varepsilon^{2}\right), \quad K^{\prime}=\frac{\pi}{2}\left(1+2 \sum_{n=1}^{\infty} q^{n^{2}}\right)^{2},
$$

where $\varepsilon \equiv 1-k^{2}$ (see Ref. [41]). Hence

$$
\frac{\pi^{2}}{4 K^{\prime 2}}=1-8 q+O\left(q^{2}\right)=1-\frac{\varepsilon}{2}+O\left(\varepsilon^{2}\right),
$$

\footnotetext{
${ }^{3}$ We shall say that $f(x)=O\left(x^{n}\right)$ as $x \rightarrow 0$ or $x \rightarrow \infty$ if $\left|x^{-n} f(x)\right|$ is bounded in this limit.
} 
and therefore

$$
\begin{aligned}
\operatorname{sn}^{-2}(2 l K / N)= & \frac{2}{3}-\frac{\varepsilon}{3}+4\left(1-\frac{\varepsilon}{2}+O\left(\varepsilon^{2}\right)\right)\left(\frac{1}{12}+q^{2 / N} \frac{q^{2(l-1) / N}}{\left(1-q^{2 l / N}\right)^{2}}\right. \\
& \left.+q^{2 / N} \sum_{j=1}^{\infty} \frac{j q^{2[j(N-l)-1] / N}\left(1-q^{2 j l / N}\right)^{2}}{1-q^{2 j}}\right) \\
= & 1+4\left(\frac{\varepsilon}{16}\right)^{2 / N}\left(\delta_{l, 1}+\delta_{l, N-1}\right)+O\left(\varepsilon^{4 / N}\right),
\end{aligned}
$$

where we have dropped an $O(\varepsilon)$ term negligible compared to $\varepsilon^{4 / N}$ for $N>4$. Thus

$$
\mathcal{H}=\mathcal{H}_{1}+\left(\frac{\varepsilon}{16}\right)^{2 / N} \mathcal{H}_{\mathrm{He}}+O\left(\varepsilon^{4 / N}\right),
$$

where

$$
\mathcal{H}_{\mathrm{He}}=2 \sum_{i}\left(1-S_{i, i+1}\right), \quad S_{N, N+1} \equiv S_{1 N} .
$$

As is well known [42], the spin permutation operators $S_{i j}$ can be expressed in terms of the su(m) Hermitian generators $J_{k}^{\alpha}$ at site $k$ (with the normalization $\operatorname{tr}\left(J_{k}^{\alpha} J_{k}^{\beta}\right)=2 \delta^{\alpha \beta}$ ) as

$$
S_{i j}=\frac{1}{m}+\frac{1}{2} \sum_{\alpha=1}^{m^{2}-1} J_{i}^{\alpha} J_{j}^{\alpha}
$$

Thus, for $m=2$ the operator $\mathcal{H}_{\mathrm{He}}$ is essentially the Hamiltonian of the spin $1 / 2$ (closed) Heisenberg chain.

\subsection{The chain sites}

We shall now prove that the chain sites of the spin chain associated with the elliptic spin Calogero-Sutherland model (2.3) are indeed the points $(2.77$, so that Eq. 2.87 is essentially equivalent to Inozemtsev's original definition. As we have seen above, it suffices to show that the scalar potential $U$ has a unique minimum (up to a rigid translation) in the Weyl alcove (2.5), with coordinates given by Eq. 2.7]. To this end, we shall first prove the following more general result:

Theorem 1. Let $f$ be an odd differentiable function with no zeros in the interval $(0,2 K)$, satisfying

$$
f(x+2 K)=-f(x), \quad \forall x .
$$

Then the point $\xi=\left(\xi_{1}, \ldots, \xi_{N}\right)$ with coordinates (2.7) is a critical point of the scalar potential

$$
U(\mathbf{x})=\sum_{1 \leqslant i \neq j \leqslant N} f^{-2}\left(x_{i}-x_{j}\right) .
$$

Proof. Note, first of all, that from Eq. 2.16 it follows that $f^{2}$ is $2 K$-periodic. Moreover, differentiating the latter equation we obtain

$$
f^{\prime}(x+2 K)=-f^{\prime}(x), \quad \forall x,
$$


and since $f^{\prime}$ is even we have

$$
f^{\prime}(K)=-f^{\prime}(-K)=-f^{\prime}(K) \quad \Longrightarrow \quad f^{\prime}(K)=0 .
$$

Let $g=-2 f^{\prime} / f^{3}$ denote the derivative of $f^{-2}$, which is defined everywhere except at even multiples of $K$. By the previous remarks, $g$ is an odd, $2 K$-periodic function satisfying

$$
g(K)=0 .
$$

We must prove that

$$
\frac{1}{2} \frac{\partial U}{\partial x_{i}}(\boldsymbol{\xi})=\sum_{j ; j \neq i} g\left(\xi_{i}-\xi_{j}\right)=\sum_{j ; j \neq i} g\left(2(i-j) \frac{K}{N}\right)=0, \quad i=1, \ldots, N .
$$

Calling $l=i-j$ we have

$$
\begin{aligned}
\sum_{j ; j \neq i} g\left(2(i-j) \frac{K}{N}\right) & =\sum_{l=1}^{i-1} g\left(\frac{2 l K}{N}\right)+\sum_{l=i-N}^{-1} g\left(\frac{2 l K}{N}\right)=\sum_{l=1}^{i-1} g\left(\frac{2 l K}{N}\right)+\sum_{l=i-N}^{-1} g\left(\frac{2(l+N) K}{N}\right) \\
& =\sum_{l=1}^{i-1} g\left(\frac{2 l K}{N}\right)+\sum_{l=i}^{N-1} g\left(\frac{2 l K}{N}\right)=\sum_{l=1}^{N-1} g\left(\frac{2 l K}{N}\right),
\end{aligned}
$$

where in the second equality we have used the $2 K$-periodicity of $g$. Thus the $N$ equations 2.19 are equivalent to the single condition

$$
\sum_{l=1}^{N-1} g\left(\frac{2 l K}{N}\right)=0 .
$$

In order to establish the previous identity, note that for arbitrary $g$ we have

$$
\sum_{l=1}^{N-1} g\left(\frac{2 l K}{N}\right)=\sum_{l=1}^{[(N-1) / 2]}\left[g\left(\frac{2 l K}{N}\right)+g\left(\frac{2(N-l) K}{N}\right)\right]+(1-\pi(N)) g(K),
$$

where $\pi(N)$ is the parity of $N$. However, in this case the last term can be omitted on account of Eq. 2.18, while

$$
g\left(\frac{2(N-l) K}{N}\right)=g\left(2 K-\frac{2 l K}{N}\right)=g\left(-\frac{2 l K}{N}\right)=-g\left(\frac{2 l K}{N}\right),
$$

so that the sum in the right-hand side of Eq. 2.21) also vanishes. This proves Eq. 2.20) and hence establishes our claim.

The previous theorem with $f(x)=\operatorname{sn} x$ implies that the point $\boldsymbol{\xi}$ with coordinates 2.7) is a critical point of the potential $U$, which obviously lies in the open region 2.5. It is not clear, however, whether this is the only critical point of $U$ in the latter region, and whether it is indeed a minimum. It turns out that both of these facts can be established in a rather straightforward way for a large class of potentials of the form 2.17). We must first deal with a technical complication stemming from the fact that $U$ is invariant under a rigid translation of the particles, i.e,

$$
U(\mathbf{x}+\lambda \mathbf{v})=U(\mathbf{x}), \quad \forall \lambda \in \mathbb{R}, \quad \mathbf{v}=(1, \ldots, 1) .
$$

This problem is addressed in Appendix B, where several standard criteria are generalized to this type of functions. With the help of these criteria, it is straightforward to obtain the following general result: 
Theorem 2. Let $U(\mathbf{x})$ be defined by equation 2.17), where $f$ is as in Theorem 1 and assume furthermore that $f$ is of class $C^{2}$ and

$$
3 f^{\prime 2}(x)-f(x) f^{\prime \prime}(x)>0, \quad \forall x \in(0,2 K) .
$$

Then the point $\boldsymbol{\xi}$ with coordinates (2.7) is the unique critical point of $U$ in the set A modulo rigid translations of the particles, and is in fact the global minimum of $U$ in the latter set.

Proof. Note, first of all, that the set $A$ in Eq. 2.5 is obviously convex and invariant under an overall translation of the particles' coordinates. Moreover, since $0<\left|x_{i}-x_{j}\right|<2 K$ for all $\mathbf{x} \in A$, the function $U$ is of class $C^{2}$ in $A$. In order to apply Lemma 2, we must evaluate the Hessian of $U$ at an arbitrary point of $A$. Calling again $g=\left(f^{-2}\right)^{\prime}$ we easily obtain

$$
\frac{\partial^{2} U}{\partial x_{i}^{2}}=2 \sum_{j ; j \neq i} g^{\prime}\left(x_{i}-x_{j}\right), \quad \frac{\partial^{2} U}{\partial x_{i} \partial x_{j}}=-2 g^{\prime}\left(x_{i}-x_{j}\right) \quad(i \neq j),
$$

where we have taken into account that $f^{2}$ is an even function. If $\mathbf{h} \in \mathbb{R}^{N}$ is an arbitrary vector we therefore have

$$
\left(\mathbf{h}, D^{2} U(\mathbf{x}) \cdot \mathbf{h}\right)=2 \sum_{i \neq j} g^{\prime}\left(x_{i}-x_{j}\right)\left(h_{i}^{2}-h_{i} h_{j}\right) .
$$

However, the function $g^{\prime} \equiv\left(f^{-2}\right)^{\prime \prime}$ is even (cf. Theorem 1 , so that

$$
\sum_{i \neq j} g^{\prime}\left(x_{i}-x_{j}\right) h_{i}^{2}=\frac{1}{2} \sum_{i \neq j} g^{\prime}\left(x_{i}-x_{j}\right)\left(h_{i}^{2}+h_{j}^{2}\right),
$$

and therefore

$$
\left(\mathbf{h}, D^{2} U(\mathbf{x}) \cdot \mathbf{h}\right)=\sum_{i \neq j} g^{\prime}\left(x_{i}-x_{j}\right)\left(h_{i}-h_{j}\right)^{2} .
$$

Since

$$
g^{\prime}=\left(f^{-2}\right)^{\prime \prime}=\frac{2}{f^{4}}\left(3 f^{\prime 2}-f f^{\prime \prime}\right)
$$

is $2 K$-periodic, by Eq. 2.22) it is positive everywhere except at even multiples of $K$. Therefore the Hessian $D^{2} U(\mathbf{x})$ is positive semidefinite, and moreover

$$
\left(\mathbf{h}, D^{2} U(\mathbf{x}) \cdot \mathbf{h}\right)=0 \quad \Longleftrightarrow \quad h_{i}=h_{j}, \forall i \neq j \quad \Longleftrightarrow \mathbf{h} \in \mathbb{R} \mathbf{v},
$$

with $\mathbf{v}=(1, \ldots, 1)$. The statement of the theorem then follows directly from Lemma 2 have

In the particular case of the potential $U$ in Eq. 2.6, for which $f(x)=\operatorname{sn} x$ by Eq. 2.17), we

$$
3 f^{\prime 2}(x)-f(x) f^{\prime \prime}(x)=k^{2} \mathrm{cn}^{4} x+2 \mathrm{cn}^{2} x+k^{\prime 2}>0, \quad \forall x \in \mathbb{R} .
$$

By the previous theorem, $U$ has a unique critical point $\xi \in A$ (modulo a rigid translation), whose coordinates are given by Eq. (2.7). Moreover, this point is the global minimum of $U$ in the open set $A$, as claimed. 


\section{Level density}

A well-known property shared by all spin chains of HS type is the fact that their level density becomes asymptotically Gaussian when the number of spins is large enough. This property has been rigorously established for all HS chains of type $A_{N-1}$ [38], and there is strong numerical evidence that it also holds for other root systems and in the supersymmetric case [43-49]. Since the Haldane-Shastry chain is the $k \rightarrow 0$ limit of Inozemtsev's chain, it is natural to investigate whether the level density of the latter chain is also approximately Gaussian when $N \gg 1$. A fundamental difficulty that must be faced in this case is the lack of a closed-form expression for the spectrum, so that one is forced to diagonalize numerically the Hamiltonian $\mathcal{H}$ in Eq. (2.8). If the symmetries of $\mathcal{H}$ are not taken into account, this approach becomes unfeasible on a standard desktop computer for $N \gtrsim 15$ spins in the most favorable case $m=2$. Fortunately, the fact that $\mathcal{H}$ depends only on spin permutation operators implies that it leaves invariant all the subspaces with well-defined "spin content". More precisely, we shall say that two basic states $|\mathbf{s}\rangle,\left|\mathbf{s}^{\prime}\right\rangle$ have the same spin content if their spin components are related by a suitable permutation. From this definition, it immediately follows that each linear subspace of $\Sigma$ spanned by all basic states with the same spin content is invariant under the permutation operators $S_{i j}$, and hence under $\mathcal{H}$.

Diagonalizing the restriction of $\mathcal{H}$ to each of these invariant subspaces we have been able to compute the whole spectrum for up to $N=18$ spins $(m=2)$ and $N=12(m=3)$, for several values of the modulus $k$. Our numerical results clearly indicate that for sufficiently large $N$ the level density of Inozemtsev's elliptic chain follows with great accuracy a Gaussian distribution with parameters $\mu$ and $\sigma$ equal to the mean and standard deviation of the spectrum. For instance, in Fig. 1 1 we have plotted the quantiles of the Gaussian distribution with parameters $\mu$ and $\sigma$ computed from the spectrum versus the corresponding quantiles of the level density of Inozemtsev's chain in the cases $N=18, m=2$ and $N=12, m=3$. It is apparent that in both cases the curve thus obtained practically coincides with the straight line $y=x$ for a wide range of energies. In fact, these plots are very similar to the corresponding one for the Haldane-Shastry chain with $N=18, m=2$, whose level density has been rigorously shown to be asymptotically Gaussian when the number of sites tends to infinity [38]. In contrast, the analogous $q-q$ plot for the Heisenberg chain with $N=18$ spins, also shown in Fig. 1. deviates more markedly from the line $y=x$. The above remarks are quantitatively corroborated by the numerical data summarized in Table 1 . where we have listed the RMS error of the fit of the spectrum's cumulative level density to the cumulative Gaussian distribution

$$
G(E)=\frac{1}{2}\left[1+\operatorname{erf}\left(\frac{E-\mu}{\sqrt{2} \sigma}\right)\right],
$$

where $\mu$ and $\sigma$ denote respectively the mean and the standard deviation of the spectrum.

\subsection{Exact formulas for the mean and variance of the energy}

Once it has been established that for sufficiently large $N$ the level density of Inozemtsev's elliptic chain is well approximated by a Gaussian distribution with parameters $\mu$ and $\sigma$ taken from the spectrum, it is of interest to evaluate the latter parameters explicitly. Although the spectrum of Inozemtsev's chain has not been computed exactly, we shall next see that it is possible to obtain a closed-form expression for the mean and the variance of its levels as functions of the number of sites $N$, the number of internal degrees of freedom $m$ and the modulus $k$. Using this expression, we shall show that (for $0<k<1$ ) $\mu$ and $\sigma^{2}$ grow as $N^{3}$ and $N^{5}$, respectively, just as is the case for the Haldane-Shastry chain [5]. 

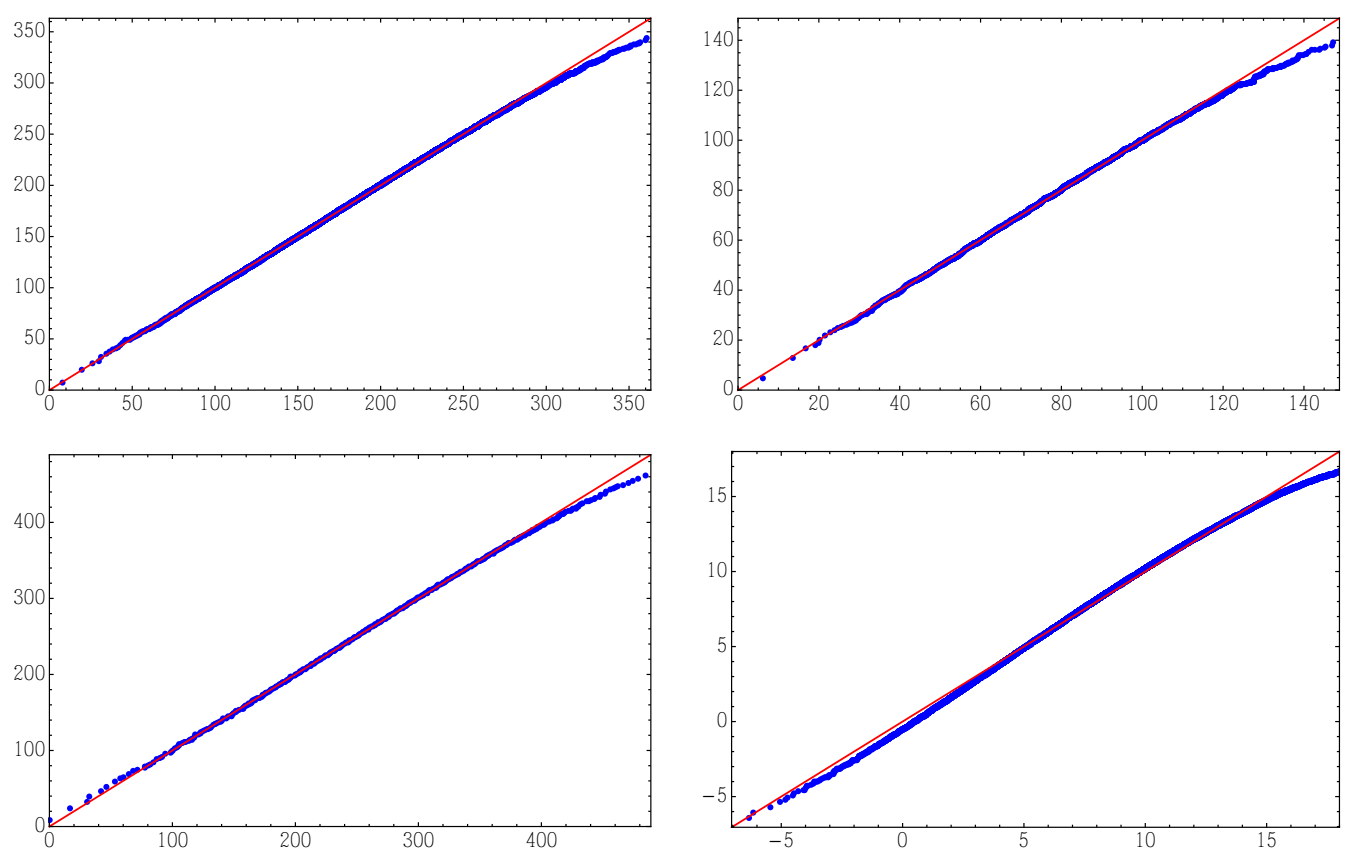

Figure 1: $q-q$ plots of the Gaussian distribution (with parameters $\mu$ and $\sigma$ computed from the spectrum) vs. the level density of Inozemtsev's chain for $N=18, m=2$ (top left) and $N=12, m=3$ (top right), where in both cases $k^{2}=1 / 2$. For comparison purposes, we have also shown the corresponding $q-q$ plots for the Haldane-Shastry chain with $N=18$, $m=2$ (bottom left) and the Heisenberg chain with $N=18$ spins (bottom right). In all four graphs, the straight line $y=x$ has been represented in red.

Let us begin with the mean energy

$$
\mu=m^{-N} \operatorname{tr} \mathcal{H}=\frac{1}{4 m^{N}} \sum_{i \neq j} h_{i j} \operatorname{tr}\left(1-S_{i j}\right),
$$

where

$$
h_{i j}=\operatorname{sn}^{-2}\left(2(i-j) \frac{K}{N}\right) \equiv h(i-j) .
$$

Clearly $\operatorname{tr} S_{i j}=m^{N-1}$, and therefore

$$
\mu=\frac{m-1}{4 m} \sum_{i \neq j} h_{i j} .
$$

In order to simplify the latter sum, we note that the function $h(z) \equiv \operatorname{sn}^{-2}(2 K z / N)$ satisfies

$$
h(z)=h(-z)=h(N-z), \quad z \in \mathbb{C},
$$

and therefore

$$
\sum_{i \neq j} h_{i j}=\sum_{i \neq j} h(i-j)=2 \sum_{l=1}^{N-1}(N-l) h(l) .
$$




\begin{tabular}{|c|c|c|c|c|}
\hline Spin chain & $N$ & $m$ & $k^{2}$ & RMS error \\
\hline \hline \multirow{4}{*}{ Inozemtsev } & \multirow{2}{*}{18} & \multirow{2}{*}{2} & 0.2 & $2.707 \times 10^{-3}$ \\
\cline { 4 - 5 } & & & 0.5 & $2.776 \times 10^{-3}$ \\
\cline { 4 - 5 } & & 0.8 & $3.594 \times 10^{-3}$ \\
\cline { 4 - 5 } & 12 & 3 & 0.5 & $4.005 \times 10^{-3}$ \\
\hline \multirow{2}{*}{ HS } & 18 & \multirow{2}{*}{2} & $(0)$ & $2.650 \times 10^{-3}$ \\
\cline { 2 - 3 } & 50 & & & $5.103 \times 10^{-4}$ \\
\hline Heisenberg & 18 & 2 & - & $1.239 \times 10^{-2}$ \\
\hline
\end{tabular}

Table 1: Root mean square error of the fit of the cumulative level density of the Inozemtsev, Haldane-Shastry and Heisenberg chains to a cumulative Gaussian distribution with parameters $\mu$ and $\sigma$ taken from the spectrum.

On the other hand, performing the change of index $l \mapsto N-l$ and using the identity (3.2) we easily obtain

$$
\sum_{l=1}^{N-1}(N-l) h(l)=\sum_{l=1}^{N-1} l h(l) \quad \Longrightarrow \quad 2 \sum_{l=1}^{N-1} \ln (l)=N \sum_{l=1}^{N-1} h(l),
$$

so that

$$
\sum_{i \neq j} h_{i j}=N \sum_{l=1}^{N-1} h(l)
$$

Thus the mean energy per site is given by

$$
\frac{\mu}{N}=\frac{m-1}{4 m} \sum_{l=1}^{N-1} h(l)
$$

Likewise, the variance of the energy can be expressed as

$$
\sigma^{2}=m^{-N} \operatorname{tr}\left[(\mathcal{H}-\mu)^{2}\right]=m^{-N} \operatorname{tr}\left[\left(\mathcal{H}^{\prime}-\mu^{\prime}\right)^{2}\right]=m^{-N} \operatorname{tr}\left(\mathcal{H}^{\prime 2}\right)-\mu^{\prime 2},
$$

where

$$
\mathcal{H}^{\prime}=\frac{1}{4} \sum_{i \neq j} h_{i j} S_{i j}
$$

and

$$
\mu^{\prime}=m^{-N} \operatorname{tr} \mathcal{H}^{\prime}=\frac{1}{4 m} \sum_{i \neq j} h_{i j}=\frac{N}{4 m} \sum_{l=1}^{N-1} h(l) .
$$

The trace of $\mathcal{H}^{\prime 2}$ can be easily computed by noting that

$$
\operatorname{tr}\left(S_{i j} S_{l n}\right)= \begin{cases}m^{N}, & \{i, j\}=\{l, n\} \\ m^{N-2}, & \text { otherwise }\end{cases}
$$

We thus have

$$
16 m^{-N} \operatorname{tr}\left(\mathcal{H}^{\prime 2}\right)=m^{-N} \sum_{i \neq j, k \neq l} h_{i j} h_{k l} \operatorname{tr}\left(S_{i j} S_{k l}\right)=m^{-2}\left(\sum_{i \neq j} h_{i j}\right)^{2}+2\left(1-m^{-2}\right) \sum_{i \neq j} h_{i j}^{2},
$$


whence, using Eqs. (3.3) (with $h$ replaced by $h^{2}$ ) and (3.6), we finally obtain

$$
\frac{\sigma^{2}}{N}=\frac{m^{2}-1}{8 N m^{2}} \sum_{i \neq j} h_{i j}^{2}=\frac{m^{2}-1}{8 m^{2}} \sum_{l=1}^{N-1} h(l)^{2} .
$$

It should be noted that Eqs. (3.4) and (3.7) are actually valid for any spin chain of the form

$$
\mathcal{H}=\frac{1}{4} \sum_{i \neq j} h_{i j}\left(1-S_{i j}\right)
$$

with $h_{i j}=h(i-j)$, provided only that the function $h$ satisfies the identities 3.2 .

By Eqs. (3.4) and (3.7), the mean and variance of the energy are proportional to the sums

$$
S_{p} \equiv \sum_{j=1}^{N-1} h(j)^{p} \equiv \sum_{j=1}^{N-1} \mathrm{sn}^{-2 p}\left(\frac{2 j K}{N}\right)
$$

with $p=1,2$. In the trigonometric case ( $k=0$ ), these sums have been evaluated in closed form (in terms of Bernoulli numbers) by Berndt and Yeap [39]. They can be easily computed for arbitrary $k \in[0,1]$ using the results in Appendix $\mathrm{C}$, as we shall next show.

Consider, to begin with, the sum $S_{1}$. Using Eqs. (2.9) and (C.4) with

$$
\omega_{3}=\frac{\mathrm{i} N K^{\prime}}{2 K} \equiv \frac{\mathrm{i} N \tau}{2}
$$

we easily obtain

$$
S_{1}=2\left(\frac{N}{2 K}\right)^{2}\left[\eta_{1}(1 / 2, \mathrm{i} N \tau / 2)-\eta_{1}(N / 2, \mathrm{i} N \tau / 2)\right]+\frac{1}{3}(N-1)\left(1+k^{2}\right),
$$

where $\eta_{1}$ is defined in Eq. A.3. From the homogeneity relation

$$
\eta_{1}(N / 2, \mathrm{i} N \tau / 2)=\frac{2 K}{N} \eta_{1}\left(K, \mathrm{i} K^{\prime}\right)
$$

(cf. Eq. [A.4]) and the well-known identity [50, Eq. 18.9.13]

$$
\eta_{1}\left(K, \mathrm{i} K^{\prime}\right)=E-\frac{1}{3}\left(2-k^{2}\right) K,
$$

where

$$
E(k)=\int_{0}^{\pi / 2} \sqrt{1-k^{2} \sin ^{2} \varphi} \mathrm{d} \varphi
$$

is the complete elliptic integral of the second kind, we finally obtain

$$
S_{1}=\frac{N^{2}}{2 K^{2}} \eta_{1}(1 / 2, \mathrm{i} N \tau / 2)+N\left(1-\frac{E}{K}\right)-\frac{1}{3}\left(1+k^{2}\right) .
$$

Likewise, from Eqs. 2.9], 3.10), (C.4), (C.6) (with $\omega_{3}=\mathrm{i} N \tau / 2$ ), and the identity

$$
\left(\frac{N}{2 K}\right)^{4} g_{2}(N / 2, \mathrm{i} N \tau / 2)=g_{2}\left(K, \mathrm{i} K^{\prime}\right)=\frac{4}{3}\left(k^{4}-k^{2}+1\right)
$$


(cf. Appendix A] and [50, Eq. 18.9.4]), after a straightforward calculation we obtain

$$
\begin{aligned}
S_{2}= & \frac{1}{960}\left(\frac{N}{K}\right)^{4} g_{2}(1 / 2, \mathrm{i} N \tau / 2)+\frac{1}{3}\left(1+k^{2}\right)\left(\frac{N}{K}\right)^{2} \eta_{1}(1 / 2, \mathrm{i} N \tau / 2)+\frac{N}{3}\left(2+k^{2}-2\left(1+k^{2}\right) \frac{E}{K}\right) \\
& -\frac{1}{45}\left(11 k^{4}+4 k^{2}+11\right) .
\end{aligned}
$$

Substituting Eqs. 3.11-3.12 into 3.4-3.7 we finally obtain the following closed-form expressions for the mean $\mu$ and the variance $\sigma^{2}$ of the energy per site of the su( $\left.m\right)$ Inozemtsev chain (2.8):

$$
\begin{gathered}
\frac{\mu}{N}=\frac{m-1}{4 m}\left[\frac{N^{2}}{2 K^{2}} \eta_{1}(1 / 2, \mathrm{i} N \tau / 2)+N\left(1-\frac{E}{K}\right)-\frac{1}{3}\left(1+k^{2}\right)\right] \\
\frac{\sigma^{2}}{N}=\frac{m^{2}-1}{8 m^{2}}\left[\frac{1}{960}\left(\frac{N}{K}\right)^{4} g_{2}(1 / 2, \mathrm{i} N \tau / 2)+\frac{1}{3}\left(1+k^{2}\right)\left(\frac{N}{K}\right)^{2} \eta_{1}(1 / 2, \mathrm{i} N \tau / 2)\right. \\
\left.+\frac{N}{3}\left(2+k^{2}-2\left(1+k^{2}\right) \frac{E}{K}\right)-\frac{1}{45}\left(11 k^{4}+4 k^{2}+11\right)\right] .
\end{gathered}
$$

It is instructive to check that when $k=0$ the above formulas reduce to the well-known ones for the Haldane-Shastry chain derived in Ref. [5]. To this end, we shall make use of the asymptotic expansions

$$
\eta_{1}\left(1 / 2, \omega_{3}\right)=\pi^{2}\left(\frac{1}{6}-4 \sum_{k=1}^{\infty} \sigma_{1}(k) q^{2 k}\right), \quad g_{2}\left(1 / 2, \omega_{3}\right)=20 \pi^{4}\left(\frac{1}{15}+16 \sum_{k=1}^{\infty} \sigma_{3}(k) q^{2 k}\right),
$$

where $\operatorname{Im} \omega_{3}>0, q=\mathrm{e}^{2 \pi \mathrm{i} \omega_{3}}$, and $\sigma_{r}(n)$ denotes the divisor function 4

$$
\sigma_{r}(n)=\sum_{j \mid n} j^{r}
$$

(see, respectively, [41, Eq. 23.8.5] and [51]). Thus

$$
\eta_{1}(1 / 2,+\mathrm{i} \infty)=\frac{\pi^{2}}{6}, \quad g_{2}(1 / 2,+\mathrm{i} \infty)=\frac{4}{3} \pi^{4},
$$

and therefore, taking into account that

$$
K(0)=\pi / 2, K^{\prime}(0)=+\infty \quad \Longrightarrow \tau(0)=+\infty,
$$

from Eqs. 3.13 - 3.14 with $k=0$ we readily obtain the well-know expressions for the mean and variance of the energy per site of the Haldane-Shastry chain:

$$
\frac{\mu}{N}=\frac{m-1}{12 m}\left(N^{2}-1\right), \quad \frac{\sigma^{2}}{N}=\frac{m^{2}-1}{360 m^{2}}\left(N^{2}-1\right)\left(N^{2}+11\right) .
$$

From the latter formulas it follows that for the Haldane-Shastry chain $\mu$ and $\sigma^{2}$ grow with $N$ respectively as $N^{3}$ and $N^{5}$. That this is the case for arbitrary $k \in[0,1)$ can be easily shown from the asymptotic formula (C.7) and the identity 2.9], which yield

$$
\begin{aligned}
\frac{4 m \mu}{N(m-1)} & =S_{1}=\left(\frac{N}{2 K}\right)^{2} \frac{(2 \pi)^{2}}{2 !} \frac{1}{6}+O(N)=\frac{1}{12}\left(\frac{N \pi}{K}\right)^{2}+O(N), \\
\frac{8 m^{2} \sigma^{2}}{N\left(m^{2}-1\right)} & =S_{2}=\left(\frac{N}{2 K}\right)^{4} \frac{(2 \pi)^{4}}{4 !} \frac{1}{30}+O\left(N^{3}\right)=\frac{1}{720}\left(\frac{N \pi}{K}\right)^{4}+O\left(N^{3}\right) .
\end{aligned}
$$



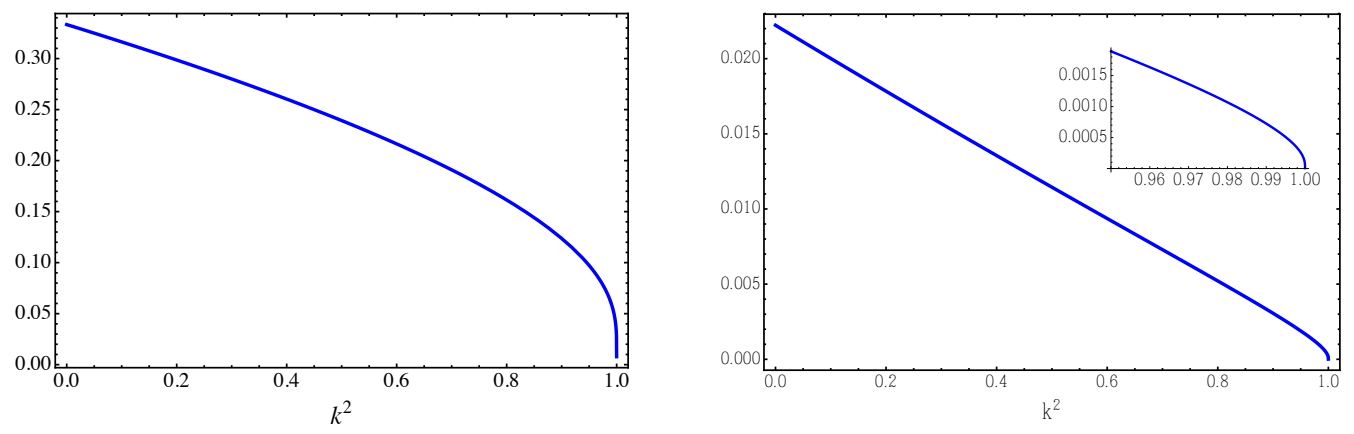

Figure 2: Coefficients of $N^{3}$ (left) and $N^{5}$ (right) in the asymptotic expansions of $4 m \mu /(m-1)$ and $8 m^{2} \sigma^{2} /\left(m^{2}-1\right)$, respectively. Inset: behavior of the latter coefficient for $0.95 \leqslant k^{2} \leqslant 1$.

Much sharper asymptotic formulas can be obtained using Eqs. (3.15) with $\omega_{3}=\mathrm{i} N \tau / 2 \rightarrow$ $+\mathrm{i} \infty$, namely

$$
\begin{aligned}
\frac{4 m \mu}{N(m-1)}=\frac{1}{12}\left(\frac{N \pi}{K}\right)^{2}+ & N\left(1-\frac{E}{K}\right)-\frac{1}{3}\left(1+k^{2}\right)+O\left(N^{2} \mathrm{e}^{-2 N \pi \tau}\right), \\
\frac{8 m^{2} \sigma^{2}}{N\left(m^{2}-1\right)}=\frac{1}{720}\left(\frac{N \pi}{K}\right)^{4} & +\frac{1}{18}\left(1+k^{2}\right)\left(\frac{N \pi}{K}\right)^{2}+\frac{N}{3}\left(2+k^{2}-2\left(1+k^{2}\right) \frac{E}{K}\right) \\
& -\frac{1}{45}\left(11 k^{4}+4 k^{2}+11\right)+O\left(N^{4} \mathrm{e}^{-2 N \pi \tau}\right) .
\end{aligned}
$$

In Figure 2 we have plotted the coefficients of $N^{3}$ and $N^{5}$ in the asymptotic expansions of $4 m \mu /(m-1)$ and $8 m^{2} \sigma^{2} /\left(m^{2}-1\right)$ as functions of $k^{2}$. Since $K(1)=+\infty$, both coefficients vanish as $k \rightarrow 1$, a fact that is also apparent from the latter figure. This was to be expected, since when $k \rightarrow 1$ the Hamiltonian (2.8) tends to (3.8) with $h_{i j}=1$, so that both $4 m \mu /(m-1)$ and $8 m^{2} \sigma^{2} /\left(m^{2}-1\right)$ tend to $N(N-1)$ on account of Eqs. (3.4)-(3.7). Note, however, that the asymptotic formulas (3.17)-(3.18) and (3.19)-(3.20) are not valid when $k=1$, since $\tau$ vanishes in this case. In fact, it is straightforward to show that Eqs. (3.13)-(3.14) yield the correct values of $\mu$ and $\sigma^{2}$ in the limit $k \rightarrow 1$ with the help of the formulas

$$
\lim _{\omega_{3} \rightarrow 0}\left[\left(2 \omega_{3}\right)^{2} \eta_{1}\left(1 / 2, \omega_{3}\right)\right]=\frac{\pi^{2}}{6}, \quad \lim _{\omega_{3} \rightarrow 0}\left[\left(2 \omega_{3}\right)^{4} g_{2}\left(1 / 2, \omega_{3}\right)\right]=\frac{4}{3} \pi^{4}
$$

which can be easily derived from 3.16 using the homogeneity properties of $\eta_{1}$ and $g_{2}$ and Legendre's relation A.5.

\subsection{Skewness and excess kurtosis}

Given a probability distribution $p(E)$ with mean $\mu$ and standard deviation $\sigma$, its skewness $\gamma_{1}$ and (excess) kurtosis $\gamma_{2}$ are defined by

$$
\gamma_{1}=\frac{\left\langle(E-\mu)^{3}\right\rangle}{\sigma^{3}}, \quad \gamma_{2}=\frac{\left\langle(E-\mu)^{4}\right\rangle}{\sigma^{4}}-3,
$$

${ }^{4}$ By definition, $j \mid n$ if $n$ is a multiple of $j$. 
where the average $\langle f(E)\rangle$ is given by

$$
\langle f(E)\rangle=\int_{-\infty}^{\infty} f(E) p(E) \mathrm{d} E .
$$

Since a normal distribution with arbitrary parameters $\mu$ and $\sigma$ has zero skewness and kurtosis, a necessary condition for the level density of the Inozemtsev chain 2.8 to be asymptotically normal is the vanishing as $N \rightarrow \infty$ of the skewness and kurtosis of its spectrum, given by

$$
\gamma_{1}=\frac{\operatorname{tr}\left[(\mathcal{H}-\mu)^{3}\right]}{m^{N} \sigma^{3}}, \quad \gamma_{2}=\frac{\operatorname{tr}\left[(\mathcal{H}-\mu)^{4}\right]}{m^{N} \sigma^{4}}-3 .
$$

In the rest of this section we shall rigorously prove that this is indeed the case (cf. Eqs. (3.29) and $(3.36)$.

As a matter of fact, we shall compute the skewness and kurtosis of the spectrum of a general chain of the form (3.8), where $h_{i j}=h(i-j)$ and $h$ satisfies the identities 3.2). Note, to begin with, that we obviously have

$$
\gamma_{1}=\frac{\operatorname{tr}\left[\left(\mu^{\prime}-\mathcal{H}^{\prime}\right)^{3}\right]}{m^{N} \sigma^{3}}, \quad \gamma_{2}=\frac{\operatorname{tr}\left[\left(\mathcal{H}^{\prime}-\mu^{\prime}\right)^{4}\right]}{m^{N} \sigma^{4}}-3,
$$

where $\mathcal{H}^{\prime}$ and $\mu^{\prime}$ are respectively given by Eqs. 3.5) and 3.6. In the case of the skewness, expanding $\left(\mu^{\prime}-\mathcal{H}^{\prime}\right)^{3}$ in powers of $\mathcal{H}^{\prime}$ and taking into account that

$$
m^{-N} \operatorname{tr}\left(\mathcal{H}^{\prime 2}\right)=\mu^{\prime 2}+\sigma^{2}
$$

we easily obtain

$$
\gamma_{1}=\frac{1}{\sigma^{3}}\left(-m^{-N} \operatorname{tr}\left(\mathcal{H}^{\prime 3}\right)+3 \mu^{\prime} \sigma^{2}+\mu^{\prime 3}\right) .
$$

By Eq. 3.5, in order to evaluate the trace of $\mathcal{H}^{\prime 3}$ we must compute the trace of the product of three spin permutation operators. It is not difficult to show that

$$
\operatorname{tr}\left(S_{i j} S_{l n} S_{p q}\right)= \begin{cases}m^{N-1}, & \{i, j\}=\{l, n\} \text { or }\{i, j\}=\{p, q\} \text { or }\{l, n\}=\{p, q\} \\ & \text { or card }\{i, j, l, n, p, q\}=3 \\ m^{N-3}, & \text { otherwise }\end{cases}
$$

which after a long but straightforward calculation leads to the identity

$$
64 m^{3-N} \operatorname{tr}\left(\mathcal{H}^{\prime 3}\right)=\left(\sum_{i \neq j} h_{i j}\right)^{3}+6\left(m^{2}-1\right)\left(\sum_{i \neq j} h_{i j}^{2}\right)\left(\sum_{l \neq n} h_{l n}\right)+8\left(m^{2}-1\right)\left(\sum_{i, j, l}^{\prime} h_{i j} h_{j l} h_{l i}-\sum_{i \neq j} h_{i j}^{3}\right) .
$$

Here, as in the rest of the paper, we have denoted by $\Sigma^{\prime}$ a sum in which no pair of indices can take the same value. Combining the previous formula with Eqs. (3.6), (3.7), and (3.23) we obtain the following explicit expression for the skewness of the spectrum of a general spin chain of the form 3.8 :

$$
\gamma_{1}=\frac{m^{2}-1}{8 m^{3} \sigma^{3}}\left(\sum_{i \neq j} h_{i j}^{3}-\sum_{i, j, l}^{\prime} h_{i j} h_{j l} h_{l i}\right) .
$$


Using Eqs. (3.3) (with $h$ replaced by $h^{3}$ ) and (D.1) to rewrite the last sum we arrive at the simplified expression

$$
\gamma_{1}=\frac{m^{2}-1}{8 m^{3} \sigma^{3}} N\left(S_{3}-2 \sum_{1 \leqslant i<j \leqslant N-1} h(i) h(j) h(j-i)\right) .
$$

Although the previous formula holds for any chain of the form [3.8), the behavior of $\gamma_{1}$ when $N \rightarrow \infty$ depends on the specific properties of the function $h$. In the case of the Inozemtsev chain 2.8 the function $\operatorname{sn} x$ is monotonically increasing in the interval $[0, K]$ and is symmetric about $K$, so that

$$
h(l)=\operatorname{sn}^{-2}\left(\frac{2 l K}{N}\right) \leqslant \operatorname{sn}^{-2}\left(\frac{2 K}{N}\right), \quad 1 \leqslant l \leqslant N-1 .
$$

Hence

$$
\sum_{1 \leqslant i<j \leqslant N-1} h(i) h(j) h(j-i) \leqslant \operatorname{sn}^{-2}\left(\frac{2 K}{N}\right) \sum_{1 \leqslant i<j \leqslant N-1} h(i) h(j)=\frac{1}{2} \operatorname{sn}^{-2}\left(\frac{2 K}{N}\right)\left(S_{1}^{2}-S_{2}\right),
$$

and using this estimate in Eq. 3.25 we obtain

$$
-\frac{m^{2}-1}{8 m^{3}} \frac{N}{\sigma^{3}}\left(S_{1}^{2}-S_{2}\right) \operatorname{sn}^{-2}\left(\frac{2 K}{N}\right) \leqslant \gamma_{1} \leqslant \frac{m^{2}-1}{8 m^{3}} \frac{N S_{3}}{\sigma^{3}} .
$$

Since

$$
S_{p} \sim N^{2 p}
$$

as $N \rightarrow \infty$ by Eqs. A.11] and (C.7), and moreover

$$
\operatorname{sn}^{-2}\left(\frac{2 K}{N}\right) \sim N^{2}, \quad \sigma^{3} \sim N^{15 / 2},
$$

from 3.27 we conclude that

$$
\gamma_{1}=O\left(N^{-1 / 2}\right) \underset{N \rightarrow \infty}{\longrightarrow} 0 .
$$

In fact, our numerical calculations strongly suggest that $\gamma_{1}$ tends to zero much faster than $N^{-1 / 2}$ as $N \rightarrow \infty$, namely ${ }^{5}$

$$
\gamma_{1} \sim N^{-5 / 2} .
$$

Consider next the kurtosis $\gamma_{2}$. Expanding $\left(\mathcal{H}^{\prime}-\mu^{\prime}\right)^{4}$ in powers of $\mathcal{H}^{\prime}$ in the second Eq. 3.22 and taking into account Eq. 3.23 we obtain

$$
\gamma_{2}=\frac{1}{\sigma^{4}}\left(m^{-N} \operatorname{tr}\left(\mathcal{H}^{\prime 4}\right)+4 \gamma_{1} \mu^{\prime} \sigma^{3}-6 \mu^{\prime 2} \sigma^{2}-\mu^{\prime 4}\right)-3 .
$$

An analysis similar to the above for $\gamma_{1}$, but considerably more involved, yields the following expression for the kurtosis of the general chain 3.8:

$$
\gamma_{2}=\frac{m^{2}-1}{16 m^{4} \sigma^{4}}\left(\left(3-m^{2}\right) \sum_{i \neq j} h_{i j}^{4}-m^{2} \sum_{i, j, l}^{\prime} h_{i j}^{2} h_{j l}^{2}+2\left(m^{2}-6\right) \sum_{i, j, l}{ }^{\prime} h_{i j}^{2} h_{j l} h_{l i}+3 \sum_{i, j, l, n}{ }^{\prime} h_{i j} h_{j l} h_{l n} h_{n i}\right) .
$$

\footnotetext{
${ }^{5}$ By definition, $f(N) \sim N^{p}$ if $N^{-p} f(N)$ has a finite and non-vanishing limit as $N \rightarrow \infty$.
} 
Using Eqs. D.2 - D.4 we can rewrite this equation more compactly as

$$
\begin{aligned}
\gamma_{2}= & \frac{m^{2}-1}{16 m^{4} \sigma^{4}} N\left(3 S_{4}-m^{2} S_{2}^{2}+4\left(m^{2}-6\right) \sum_{1 \leqslant i<j \leqslant N-1} h^{2}(i) h(j) h(j-i)\right. \\
& \left.+6 \sum_{3 \leqslant i+j+l \leqslant N-1} h(i) h(j) h(l) h(i+j+l)+12 \sum_{3 \leqslant i+j+l \leqslant N-1} h(i) h(j) h(i+l) h(j+l)\right) .
\end{aligned}
$$

Each of the terms in parentheses in the latter equation is $O\left(N^{8}\right)$. Indeed, for the first two terms this is an immediate consequence of Eq. (3.28). As for the third one, we have

$$
\begin{aligned}
\sum_{1 \leqslant i<j \leqslant N-1} h^{2}(i) h(j) h(j-i) & =\frac{1}{2} \sum_{1 \leqslant i<j \leqslant N-1}\left[h^{2}(i) h(j)+h^{2}(j) h(i)\right] h(j-i) \\
& =\frac{1}{2} \sum_{1 \leqslant i \neq j \leqslant N-1} h^{2}(i) h(j) h(j-i) \leqslant \frac{1}{2} \operatorname{sn}^{-2}\left(\frac{2 K}{N}\right) \sum_{1 \leqslant i \neq j \leqslant N-1} h^{2}(i) h(j) \\
& =\frac{1}{2} \operatorname{sn}^{-2}\left(\frac{2 K}{N}\right)\left(S_{1} S_{2}-S_{3}\right)=O\left(N^{8}\right) .
\end{aligned}
$$

The second sum in Eq. (3.32) can be easily estimated by noting that

$$
\sum_{3 \leqslant i+j+l \leqslant N-1} h(i) h(j) h(l) h(i+j+l) \leqslant \mathrm{sn}^{-2}\left(\frac{2 K}{N}\right) \sum_{3 \leqslant i+j+l \leqslant N-1} h(i) h(j) h(l)
$$

and

$$
\sum_{3 \leqslant i+j+l \leqslant N-1} h(i) h(j) h(l)=\sum_{\substack{3 \leqslant i, j, l \leqslant N-1 \\ i+j+l \geqslant 2 N+1}} h(i) h(j) h(l) \Longrightarrow \sum_{3 \leqslant i+j+l \leqslant N-1} h(i) h(j) h(l) \leqslant \frac{1}{2} S_{1}^{3},
$$

so that

$$
\sum_{3 \leqslant i+j+l \leqslant N-1} h(i) h(j) h(l) h(i+j+l) \leqslant \frac{1}{2} \operatorname{sn}^{-2}\left(\frac{2 K}{N}\right) S_{1}^{3}=O\left(N^{8}\right) .
$$

Likewise,

$$
\begin{gathered}
\sum_{3 \leqslant i+j+l \leqslant N-1} h(i) h(j) h(i+l) h(j+l) \leqslant \operatorname{sn}^{-2}\left(\frac{2 K}{N}\right) \sum_{3 \leqslant i+j+l \leqslant N-1} h(i) h(j) h(i+l) \\
=\operatorname{sn}^{-2}\left(\frac{2 K}{N}\right) \sum_{\substack{1 \leqslant i, j, l \leqslant N-1 \\
i<l, j+l \leqslant N-1}} h(i) h(j) h(l) \leqslant \frac{1}{2} \operatorname{sn}^{-2}\left(\frac{2 K}{N}\right) S_{1}^{3}=O\left(N^{8}\right) .
\end{gathered}
$$

From the estimates $3.33-(3.35)$ and the fact that $\sigma^{4} \sim N^{10}$ it immediately follows that

$$
\gamma_{2}=O\left(N^{-1}\right) \underset{N \rightarrow \infty}{\longrightarrow} 0
$$

In fact, numerical evaluation of $\gamma_{2}$ for $N$ up to 300 and different values of $k^{2}$ shows that

$$
\gamma_{2} \sim N^{-1}
$$

as $N \rightarrow \infty$. 


\section{Integrability in terms of statistical properties of the spectrum}

In this section we shall study several global properties of the spectrum of the Inozemtsev chain which are of interest for analyzing the integrable versus chaotic behavior of a quantum system. As is well known, a preliminary step in the analysis of a spectrum in the context of quantum chaos is the computation of a smooth approximation to its (cumulative) level density. This is essentially due to the fact that, in order to compare different spectra (or different parts of a given spectrum), it is necessary to first normalize them so that the resulting spectra have an approximately uniform level density. More precisely, let $E_{0}<E_{1}<\cdots<E_{n}$ be the distinct energy levels of a (finite) quantum spectrum, with respective degeneracies $d_{0}, \ldots, d_{n}$. If $\varepsilon(E)$ is a smooth approximation to the cumulative level density

$$
F(E)=\frac{1}{D} \sum_{i ; E_{i} \leqslant E} d_{i}, \quad D \equiv \sum_{i=0}^{n} d_{i},
$$

it can be easily shown [52] that the level density of the "unfolded" spectrum

$$
\varepsilon_{i} \equiv \varepsilon\left(E_{i}\right), \quad i=0, \ldots, n,
$$

is approximately equal to 1 .

\subsection{Spacings distribution}

The first property that we shall consider is the distribution of normalized spacings between consecutive levels of the unfolded spectrum, given by

$$
s_{i}=\frac{n\left(\varepsilon_{i}-\varepsilon_{i-1}\right)}{\varepsilon_{n}-\varepsilon_{0}}, \quad i=1, \ldots, n \text {. }
$$

According to a well-known conjecture due to Bohigas, Giannoni and Schmit [33], in a quantum system invariant under time reversal (with integer total spin, or invariant under rotations around an axis) whose classical limit is chaotic the probability density $p(s)$ of these consecutive spacings should approximately follow Wigner's law

$$
p(s)=\frac{\pi s}{2} \mathrm{e}^{-\pi s^{2} / 4},
$$

characteristic of the Gaussian orthogonal ensemble (GOE) in random matrix theory [53]. In the integrable case, through a heuristic argument based on the semiclassical limit Berry and Tabor conjectured that the probability density $p(s)$ should be Poissonian for a generic integrable system [32]. This conjecture has been confirmed for a wide class of such systems of physical interest, including the Heisenberg chain, the Hubbard model, the $t$ - $J$ model [34] and the chiral Potts model [54]. On the other hand, in most chains of HS type (including the original Haldane-Shastry and the Polychronakos-Frahm chains) the raw spectrum is either exactly or approximately equispaced [45, 47, 55]. This fact, together with the Gaussian character of the level density, can be shown to imply [55] that the cumulative spacings distribution $P(s)=\int_{0}^{s} p(t) \mathrm{d} t$ of the whole spectrum is approximately of the form

$$
P(s)=1-\frac{2}{\sqrt{\pi} s_{\max }} \sqrt{\log \left(\frac{s_{\max }}{s}\right)} .
$$


The energies of Inozemtsev's chain 2.8 are not even approximately equispaced, so that it should not be expected that the spacings distribution of its whole spectrum obey the latter equation; in fact, it can be numerically verified that this is not the case. We shall therefore follow a more conventional approach, studying the spectrum of the restriction of the Hamiltonian to simultaneous eigenspaces of a suitable set of mutually commuting first integrals. We shall restrict ourselves to the $\mathrm{su}(2)$ case, for which these first integrals can be taken as the operators $\mathbf{S}^{2} \equiv\left(S^{x}\right)^{2}+\left(S^{y}\right)^{2}+\left(S^{z}\right)^{2}, S^{z}$ and $T$, where

$$
S^{\alpha}=\frac{1}{2} \sum_{i} \sigma_{i}^{\alpha} \quad(\alpha=x, y, z),
$$

$\sigma_{i}^{\alpha}$ denotes the Pauli matrix $\sigma^{\alpha}$ acting on the internal space of the $i$-th spin, and $T$ is the translation operator along the chain, defined by

$$
T\left|s_{1}, s_{2}, \ldots, s_{N}\right\rangle=\left|s_{2}, s_{3}, \ldots, s_{N}, s_{1}\right\rangle .
$$

Since $T$ is obviously unitary and satisfies $T^{N}=1$, its eigenvalues are the $N$ roots of unity. Thus the eigenstates of $T$ have well-defined (modulo $2 \pi$ ) total momentum $P_{\text {tot }}=2 j \pi / N$, with $j=0,1, \ldots, N-1$. Note also that in the $\mathrm{s} u(2)$ case Eq. 2.15 yields

$$
S_{i j}=\frac{1}{2}\left(1+\sigma_{i} \cdot \sigma_{j}\right), \quad \sigma_{i} \equiv\left(\sigma_{i}^{x}, \sigma_{i}^{y}, \sigma_{i}^{z}\right),
$$

from which it easily follows that the square of the total spin operator is given by

$$
\mathbf{S}^{2}=\sum_{i<j} S_{i j}-\frac{N}{4}(N-4) .
$$

We have numerically computed the cumulative distribution $P(s)$ of Inozemtsev's chain (2.8) for several values of $k$ and $N$, in subspaces with zero momentum and $S^{z}=0$ (for even $N$ ) or $S^{z}=1 / 2$ (for odd $N$ ), choosing the eigenvalue of the operator $\mathbf{S}^{2}$ so as to obtain the largest possible spectrum ${ }^{6}$ It is apparent in all cases that approximately $25 \%$ of the spacings are several orders of magnitude larger than the rest, indicating that when $0<k<1$ the highly degenerate and approximately equispaced spectrum of the Haldane-Shastry chain $(k=0)$ splits into multiple subspectra whose mutual distance is much larger than the typical spacing within each subspectrum. When this fact is taken into account by removing the largest spacings and renormalizing the remaining ones to mean 1 , the renormalized spacings distribution is very well approximated by Poisson's law (cf. Fig. 3 for $k^{2}=1 / 2$ ). Thus the spacings distribution of Inozemtsev's chain behaves as predicted by the Berry-Tabor conjecture for a "generic" integrable system like, e.g., the Heisenberg chain.

\subsection{Spectral noise}

In recent years, an alternative test for detecting quantum integrability versus chaotic behavior has been proposed by Relaño et al. [35, 36]. The test is based on the Fourier analysis of the

\footnotetext{
${ }^{6}$ Interestingly, the level density is also approximately Gaussian when the Hamiltonian is restricted to (sufficiently large) subspaces with well-defined eigenvalues of the operators $\mathbf{S}^{2}, S^{z}$ and $P_{\text {tot }}$, as is the case with the whole spectrum (cf. Section 3). Thus, in all the subspaces considered the unfolding function $\varepsilon(E)$ can be simply taken as the cumulative distribution function 3.1 of a normal distribution with parameters equal to the mean and the standard deviation of the corresponding subspectrum.
} 


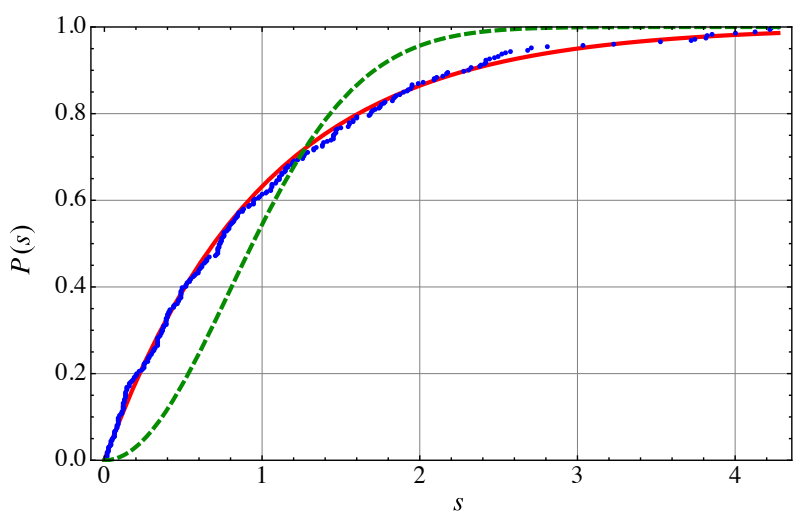

Figure 3: Cumulative spacings distribution $P(s)$ of Inozemtsev's chain with $k^{2}=1 / 2, m=2$ and $N=18$ in the subspace with total spin $S=2, S_{z}=0, P_{\text {tot }}=0$ after removing the largest $25 \%$ spacings (blue dots), compared to Poisson's law (continuous red line) and Wigner's law (dashed green line). The RMS error of the fit of $P(s)$ to Poisson's distribution is $1.33 \cdot 10^{-2}$.

fluctuations of the spacings of the unfolded spectrum from their mean (the so-called "spectral noise"). More precisely, one considers the statistic

$$
\delta_{j}=\sum_{i=1}^{j}\left(s_{i}-1\right), \quad j=1, \ldots, n,
$$

and its discrete Fourier transform

$$
\hat{\delta}_{v}=\frac{1}{\sqrt{n}} \sum_{j=1}^{n} \delta_{j} \mathrm{e}^{-2 \pi \mathrm{i} j v / n}, \quad v=1, \ldots, n .
$$

According to Ref. [36], for an integrable system the power spectrum $\mathcal{P}(v) \equiv\left|\hat{\delta}_{v}\right|^{2}$ should behave as $v^{-2}$ (blue noise) for sufficiently small values of $v$, while for a fully chaotic system $\mathcal{P}(v)$ should fall off as $v^{-1}$ (pink noise). This conjecture has been confirmed in subsequent publications for quantum billiards and several random matrix ensembles [56-58]. Again, spin chains of HS type seem to be somewhat exceptional also in this respect, since the power spectrum of both the Haldane-Shastry and the Polychronakos-Frahm chain behaves as $v^{-4}$ (black noise) for small $v$ [59].

We have evaluated numerically the power spectrum of Inozemtsev's chain with $N=18$ spins $1 / 2$ and $k^{2}=0.05,0.1, \ldots, 0.95$ in subspaces with $7 S^{z}=0$, total spin $S=1,2,3$ and $\left|P_{\text {tot }}\right|=j \pi / 9(j=1, \ldots, 8)$. For each value of $k^{2}$, we have averaged $\mathcal{P}(v)$ over the $3 \times 8=24$ subspectra under consideration, removing an appropriate number of energies when necessary to make all of them of equal length. As in the study of the spacings distribution in the previous

\footnotetext{
${ }^{7}$ Since the total momentum is defined up to an integer multiple of $2 \pi$ its possible values can be taken as $j \pi / 9$, with $j=0, \pm 1, \ldots, \pm 8,9$. The subspaces with $P_{\text {tot }}= \pm j \pi / 9$ for $j=1, \ldots, 8$ (and fixed $S^{z}, \mathbf{S}^{2}$ ) have the same energies, and can therefore be merged. The two remaining subspaces with $j=0,9$ have been discarded, since their dimension is smaller than that of the other ones roughly by a factor of 2 . Similarly, the subspaces with $S=0$ and $S=4,5, \ldots, 9$ have been dropped due to their smaller dimension.
} 
subsection, we have also dropped the largest $25 \%$ spacings in each of these subspaces prior to the computation of the power spectrum. Our analysis shows that in all cases $\mathcal{P}(v)$ is proportional to $v^{-\alpha}$ (for $1 \leqslant v \leqslant n / 4$ ), where the exponent $\alpha$ varies very slightly with $k^{2}$ between 2.097 (for $k^{2}=0.15$ ) to 2.145 (for $k^{2}=0.05$ ). For instance, for $k^{2}=1 / 2$ the exponent $\alpha$ is equal to 2.100, with coefficient of determination $r^{2}=0.9870$. For comparison purposes, we have performed a similar analysis for the su(2) Heisenberg chain with $N=18$ spins, finding that $\alpha=1.916$ with coefficient of determination $r=0.9882$ (see Fig. 4). These facts clearly suggest that Inozemtsev's chain is closer to more "standard" integrable systems like the Heisenberg chain than to the Haldane-Shastry $\left(k^{2}=0\right)$ or Polychronakos-Frahm chains, even for values of $k^{2}$ close to 0 .

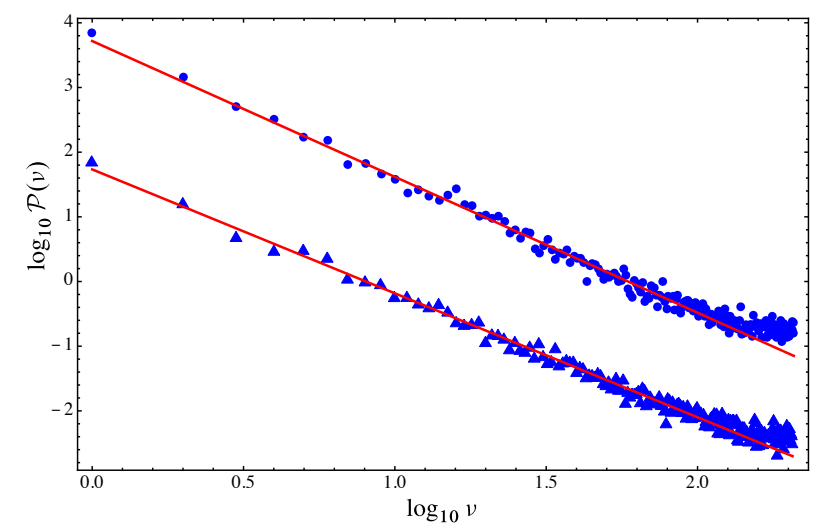

Figure 4: Blue dots: $\log -\log$ plot of $\mathcal{P}(v)$ for the spin chain 2.8 with 18 spins $1 / 2, k^{2}=0.5$ and $S^{z}=0$, computed by averaging over 24 subspectra of $n=416$ spacings with quantum numbers $S=1,2,3$ and $\left|P_{\text {tot }}\right|=\pi / 9,2 \pi / 9, \ldots, 8 \pi / 9$. Blue triangles: analogous plot for the spin $1 / 2$ Heisenberg chain 2.14 with 18 spins and $S^{z}=0$ (24 subspectra with $n=412$ spacings each, with the same quantum numbers as before). The solid red lines represent the best-fit straight lines to the data in the range $1 \leqslant v \leqslant n / 4$. (The plot for the Heisenberg chain has been lowered to avoid overlapping).

\subsection{Average degeneracy}

It is well-known that the energy spectrum of all spin chains of HS type associated with the $A_{N-1}$ root system is highly degenerate, much more so than is the case with more typical integrable models like the Heisenberg chain. For the original Haldane-Shastry and Polychronakos-Frahm chains, this high degeneracy is ultimately due to the invariance under suitable realizations of the Yangian $Y(\operatorname{su}(m))[4,60]$. An important consequence of the Yangian symmetry of these chains is their equivalence to a classical vertex model with energies given by [6]

$$
E_{\mathbf{n}}=\sum_{i=1}^{N-1} \mathcal{E}(i) \delta\left(n_{i}, n_{i+1}\right), \quad \mathbf{n} \equiv\left(n_{1}, \ldots, n_{N}\right) \in\{1, \ldots, m\}^{N},
$$

where

and

$$
\delta(i, j)= \begin{cases}1, & i>j \\ 0, & i \leqslant j\end{cases}
$$

$$
\mathcal{E}(i)= \begin{cases}i(N-i), & \text { for the HS chain } \\ i, & \text { for the PF chain } .\end{cases}
$$


In fact, Eq. (4.3) also holds for the Frahm-Inozemtsev (hyperbolic) chain [16] with dispersion relation $\mathcal{E}(i)=i(\alpha+i-1)$, where $\alpha$ is the positive parameter appearing in this model [6, 59]. In view of these facts, it is natural to enquire whether the elliptic chain (2.8) is equivalent to a vertex model (4.3)-(4.4) for a suitable choice of the dispersion relation $\mathcal{E}(i)$. As it turns out, the number of distinct levels $\ell \equiv \ell(N, m)$ of the model (4.3)-(4.4) admits an upper bound which is independent of the dispersion relation $\mathcal{E}(i)$. More precisely, it can be shown [61] that

$$
\ell \leqslant F_{N+m-1}^{(m)},
$$

where $F_{n}^{(m)}$ is the $n$-th $m$-Fibonacci number [62] defined by $F_{0}^{(m)}=\cdots=F_{m-2}^{(m)}=0, F_{m-1}^{(m)}=1$, and

$$
F_{n}^{(m)}=\sum_{j=1}^{m} F_{n-j}^{(m)}, \quad n \geqslant m .
$$

Equivalently, the average degeneracy $\bar{d} \equiv m^{N} / \ell$ of the model (4.3)-4.4) satisfies the inequality

$$
\bar{d} \geqslant \frac{m^{N}}{F_{N+m-1}^{(m)}},
$$

regardless of the functional form of the dispersion relation. In particular, a necessary condition for the $s u(m)$ Inozemtsev chain to be equivalent to a vertex model of the form (4.3)- $(4.4)$ is that its average degeneracy $\bar{d}$ satisfy the inequality (4.7). In order to test this fact in the simplest case $m=2$, we have numerically computed the average degeneracy of the su(2) chain $(2.8)$ for $10 \leqslant N \leqslant 18$ and $k^{2}=0.1,0.2, \ldots, 0.9$. In fact, for these values of $k^{2}$ we have found that $\bar{d}$ is independent of $k^{2}$ (up to small numerical fluctuations), and thus can be regarded as a function of $N$ only. Our calculations clearly show that the function $\bar{d}(N)$ is definitely smaller than the RHS of Eq. 4.7) in the range under consideration; cf. Fig. 5. Thus for $10 \leqslant N \leqslant 18$ the elliptic chain (2.8) cannot be equivalent to a vertex model of the form 4.3)-4.4. Moreover, from Fig. 5 it is also apparent that the RHS of Eq. 4.7) grows much faster with $N$ than the elliptic chain's average degeneracy $\bar{d}(N)$. This strongly suggests that Inozemtsev's chain is not equivalent to any vertex model (4.3)-4.4 for arbitrary $N$. This conclusion is consistent with the widespread belief that Inozemtsev's chain does not possess the Yangian symmetry for finite values of $N$. As a further confirmation of this assertion, we have numerically verified that for $10 \leqslant N \leqslant 18$ the average degeneracy of Inozemtsev's su(2) chain essentially coincides with that of the spin $1 / 2$ Heisenberg chain 2.14, which is known to be invariant under the Yangian only in an infinite lattice [37] (see again Fig. 5].

\section{Acknowledgments}

This work was supported in part by Spain's MINECO under grant no. FIS2011-22566. The authors would like to thank Vladimir Inozemtsev for useful discussions on the elliptic chain's integrability and Armando Relaño for enlightening conversations on quantum chaos.

\section{Appendix A. Some useful identities relating Weierstrass and Jacobi elliptic functions}

Let $\wp\left(z ; \omega_{1}, \omega_{3}\right)$ denote the Weierstrass's elliptic function [63] with fundamental half-periods $\omega_{1}$ and $\omega_{3}\left(\right.$ where $\left.\operatorname{Im}\left(\omega_{3} / \omega_{1}\right)>0\right)$, defined by

$$
\wp\left(z ; \omega_{1}, \omega_{3}\right)=\frac{1}{z^{2}}+\sum_{(l, n) \in \mathbb{Z}^{2} \backslash\{0\}}\left[\frac{1}{\left(z-2 l \omega_{1}-2 n \omega_{3}\right)^{2}}-\frac{1}{\left(2 l \omega_{1}+2 n \omega_{3}\right)^{2}}\right] .
$$




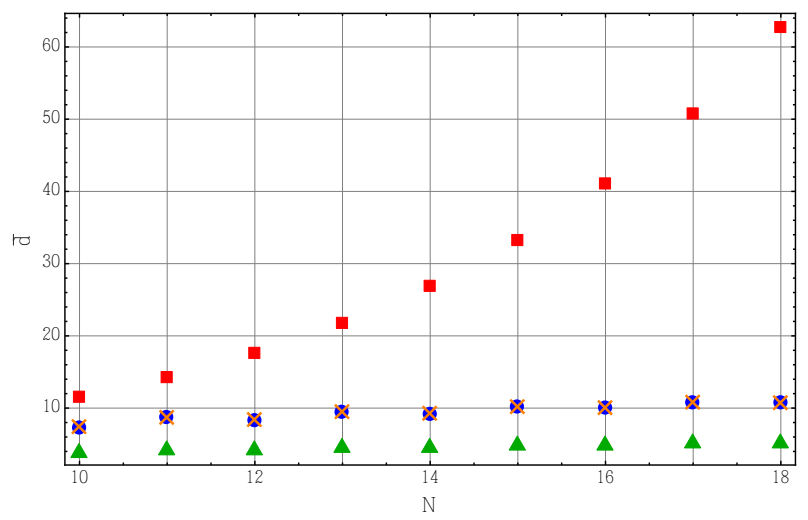

Figure 5: Average degeneracy $\bar{d}$ of Inozemtsev's su(2) chain (blue dots) as a function of the number of spins $N$, vs. the minimum average degeneracy of a vertex model of the form (4.3)-(4.4), given by the RHS of Eq. (4.7) (red squares). For comparison purposes, we have also plotted the average degeneracy of the su(2) Heisenberg chain (orange crosses) and of a rotationally and translationally invariant spin chain 3.8 with random interactions (green triangles).

We shall adopt the abbreviated notation $\wp(z)$ when the periods of $\wp$ are clear from the context. The function $\wp$ is even, doubly periodic and meromorphic, with a double pole on the sites of the period lattice $2 l \omega_{1}+2 n \omega_{3}(l, n \in \mathbb{Z})$. From the previous formula it is obvious that $\wp$ is real on the real axis when $\omega_{1}$ is real and $\omega_{3}$ is pure imaginary, and that it satisfies the homogeneity relation

$$
\wp\left(\lambda z ; \lambda \omega_{1}, \lambda \omega_{3}\right)=\lambda^{-2} \wp\left(z ; \omega_{1}, \omega_{3}\right) .
$$

The function $\wp$ is minus the derivative of the Weierstrass $\zeta$ function defined by

$$
\zeta(z) \equiv \zeta\left(z ; \omega_{1}, \omega_{3}\right)=\frac{1}{z}+\sum_{(l, n) \in \mathbb{Z}^{2} \backslash\{0\}}\left[\frac{1}{z-2 l \omega_{1}-2 n \omega_{3}}+\frac{1}{2 l \omega_{1}+2 n \omega_{3}}+\frac{z}{\left(2 l \omega_{1}+2 n \omega_{3}\right)^{2}}\right],
$$

which is an odd meromorphic function with simple poles on the lattice $2 l \omega_{1}+2 n \omega_{3}(l, n \in \mathbb{Z})$. Note, however, that $\zeta$ is not $\left(2 \omega_{1}, 2 \omega_{3}\right)$-periodic, but rather verifies

$$
\zeta\left(z+2 \omega_{i}\right)=\zeta(z)+2 \eta_{i}
$$

where

$$
\eta_{i} \equiv \eta_{i}\left(\omega_{1}, \omega_{3}\right)=\zeta\left(\omega_{i} ; \omega_{1}, \omega_{3}\right) .
$$

The function $\zeta$ obviously satisfies the homogeneity relation

$$
\zeta\left(\lambda z ; \lambda \omega_{1}, \lambda \omega_{3}\right)=\lambda^{-1} \zeta\left(z ; \omega_{1}, \omega_{3}\right)
$$

in particular,

$$
\eta_{i}\left(\lambda \omega_{1}, \lambda \omega_{3}\right)=\lambda^{-1} \eta_{i}\left(\omega_{1}, \omega_{3}\right) .
$$

In addition, the numbers $\eta_{i}$ are related by Legendre's identity

$$
\eta_{1} \omega_{3}-\eta_{3} \omega_{1}=\frac{\mathrm{i} \pi}{2}
$$


The Laurent series of $\wp$ around the origin has the form

$$
\wp(z)=\frac{1}{z^{2}}+\frac{g_{2}}{20} z^{2}+\frac{g_{3}}{28} z^{4}+\left[z^{6}\right]
$$

where $\left[z^{6}\right]$ denotes a function analytic at the origin with a zero of order at least 6 at this point, and the invariants $g_{i} \equiv g_{i}\left(\omega_{1}, \omega_{3}\right)$ are given by

$$
g_{2}=\frac{1}{60} \sum_{(l, n) \in \mathbb{Z}^{2} \backslash\{0\}}\left(2 l \omega_{1}+2 n \omega_{3}\right)^{-4}, \quad g_{3}=\frac{1}{140} \sum_{(l, n) \in \mathbb{Z}^{2} \backslash\{0\}}\left(2 l \omega_{1}+2 n \omega_{3}\right)^{-6}
$$

These definitions obviously imply the homogeneity relations

$$
g_{2}\left(\lambda \omega_{1}, \lambda \omega_{3}\right)=\lambda^{-4} g_{2}\left(\omega_{1}, \omega_{3}\right), \quad g_{3}\left(\lambda \omega_{1}, \lambda \omega_{3}\right)=\lambda^{-6} g_{2}\left(\omega_{1}, \omega_{3}\right)
$$

As is well known, the derivative of $\wp$ is an algebraic function of $\wp$, namely

$$
\wp^{\prime 2}=4 \wp^{3}-g_{2} \wp-g_{3} .
$$

From this identity it is straightforward to show that the invariants of $\wp$ are related to the numbers

$$
e_{i}=\wp\left(\omega_{i}\right), \quad i=1,2,3,
$$

(where $\omega_{2} \equiv-\omega_{1}-\omega_{3}$ by definition) by the well-known formula

$$
4 s^{3}-g_{2} s-g_{3}=4\left(s-e_{1}\right)\left(s-e_{2}\right)\left(s-e_{3}\right) .
$$

It is important to recall that when $\omega_{1}, \mathrm{i} \omega_{3} \in \mathbb{R}$ all the numbers $e_{i}, g_{i}$ are real and satisfy the inequalities

$$
e_{1}>e_{2}>e_{3}, \quad g_{3}^{2}>27 g_{2}^{3} .
$$

We shall denote by $\operatorname{sn}(z, k)$ (or, in abbreviated fashion, sn $z$ ) Jacobi's elliptic sine with modulus $k$. Its fundamental periods are $4 K(k)$ and $2 \mathrm{i} K^{\prime}(k)$, where

$$
K(k)=\int_{0}^{\pi / 2} \frac{\mathrm{d} x}{\sqrt{1-k^{2} \sin ^{2} x}}
$$

is the complete elliptic integral of the first kind, and

$$
K^{\prime}(k)=K\left(k^{\prime}\right), \quad k^{\prime} \equiv \sqrt{1-k^{2}} .
$$

When $0 \leqslant k \leqslant 1$ the numbers $K \equiv K(k)$ and $K^{\prime} \equiv K^{\prime}(k)$ are both real (with $K(1)=K^{\prime}(0)=\infty$ ), and $\operatorname{sn} z$ is real for real values of $z$. It is also well known that for $k=0,1$ Jacobi's elliptic sine reduces to an elementary function, namely

$$
\operatorname{sn}(z, 0)=\sin x, \quad \operatorname{sn}(z, 1)=\tanh z .
$$

The elliptic sine is related to the Weierstrass $\wp$ function by the well-known identity

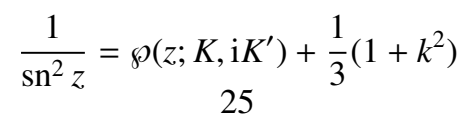


(cf. 63, Eq. 6.9.11]). Conversely, given two nonzero complex numbers $\omega_{1}, \omega_{3}$ with $\operatorname{Im}\left(\omega_{3} / \omega_{1}\right)>$ 0 it is shown in the latter reference that

$$
\wp\left(z ; \omega_{1}, \omega_{3}\right)=e_{3}+\frac{e_{1}-e_{3}}{\operatorname{sn}^{2}\left(\sqrt{e_{1}-e_{3}} z, k\right)},
$$

where the modulus of the elliptic sine is determined by

$$
k^{2}=\frac{e_{2}-e_{3}}{e_{1}-e_{3}} .
$$

In particular, when $\omega_{1}$ and $i \omega_{3}$ are both real the previous identity and the inequalities A.8 imply that $k^{2}$ is a real number in the interval $(0,1)$. Note also that from Eq. A.12 it immediately follows the important relation

$$
\frac{\omega_{3}}{\omega_{1}}=\frac{\mathrm{i} K^{\prime}}{K} \equiv \mathrm{i} \tau
$$

\section{Appendix B. Extrema of translation-invariant functions}

Lemma 1. Let $U$ be a scalar real-valued function of class $C^{2}$ in an open subset $\Omega \subset \mathbb{R}^{N}$. Suppose that there is a fixed vector $\mathbf{v} \in \mathbb{R}^{N}$ such that $\Omega$ is invariant under translations in the direction of $\mathbf{v}$, and

$$
U(\mathbf{x}+\lambda \mathbf{v})=U(\mathbf{x}), \quad \forall \mathbf{x} \in \Omega, \quad \forall \lambda \in \mathbb{R} .
$$

If $\mathbf{x}_{0} \in \Omega$ is a critical point of $U$ satisfying

$$
\left(\mathbf{h}, D^{2} U\left(\mathbf{x}_{0}\right) \cdot \mathbf{h}\right)>0, \quad \forall \mathbf{h} \in(\mathbb{R} \mathbf{v})^{\perp}, \mathbf{h} \neq 0,
$$

then $U$ has a local minimum at $\mathbf{x}_{0}$.

Proof. Simply change variables so that $\mathbf{v}$ is in the direction of a coordinate vector.

Note that, since

$$
D^{2} U(\mathbf{x}) \cdot \mathbf{v}=0,
$$

we can replace B.1p by the apparently stronger condition

$$
\left(\mathbf{h}, D^{2} U\left(\mathbf{x}_{0}\right) \cdot \mathbf{h}\right)>0, \quad \forall \mathbf{h} \notin \mathbb{R} \mathbf{v} .
$$

Lemma 2. Let $U$ and $\Omega$ be as in the previous lemma. Suppose, moreover, that $\Omega$ is convex and

$$
\left(\mathbf{h}, D^{2} U(\mathbf{x}) \cdot \mathbf{h}\right)>0, \quad \forall \mathbf{x} \in \Omega, \quad \forall \mathbf{h} \in(\mathbb{R} \mathbf{v})^{\perp}, \mathbf{h} \neq 0 .
$$

Then $U$ has at most one critical point in $\Omega$ modulo translations along the vector $\mathbf{v}$, and this critical point (if it exists at all) is necessarily a global minimum.

Proof. Note, first of all, that the condition on the Hessian of $U$ is equivalent to

$$
\left(\mathbf{h}, D^{2} U(\mathbf{x}) \cdot \mathbf{h}\right)>0, \quad \forall \mathbf{x} \in \Omega, \quad \forall \mathbf{h} \notin \mathbb{R} \mathbf{v} .
$$

Suppose, to begin with, that $\mathbf{x}_{0}, \mathbf{y}_{0}$ are critical points of $U$ in $\Omega$ such that $\mathbf{y}_{0}-\mathbf{x}_{0}$ is not proportional to $\mathbf{v}$. Since $\Omega$ is convex, the segment $t \mathbf{y}_{0}+(1-t) \mathbf{x}_{0}(0 \leqslant t \leqslant 1)$ lies in $\Omega$, so that the function

$$
\varphi(t)=U\left(t \mathbf{y}_{0}+(1-t) \mathbf{x}_{0}\right)
$$


has two critical points at $t=0,1$. By Rolle's theorem, there is a point $t_{0} \in(0,1)$ such that

$$
0=\varphi^{\prime \prime}\left(t_{0}\right)=\left(\mathbf{y}_{0}-\mathbf{x}_{0}, D^{2} U\left(t_{0} \mathbf{y}_{0}+\left(1-t_{0}\right) \mathbf{x}_{0}\right) \cdot\left(\mathbf{y}_{0}-\mathbf{x}_{0}\right)\right) .
$$

This contradicts condition (B.2), since $\mathbf{y}_{0}-\mathbf{x}_{0}$ is not proportional to $\mathbf{v}$. Thus $U$ has at most one critical point in $\Omega$, modulo translations along $\mathbf{v}$.

Let now $\mathbf{x}_{0} \in \Omega$ be a critical point of $U$, which must be a local minimum on account of the previous lemma. To prove that $\mathbf{x}_{0}$ is actually a global minimum, suppose that $U\left(\mathbf{y}_{0}\right) \leqslant U\left(\mathbf{x}_{0}\right)$ for some $\mathbf{y}_{0} \in \Omega$ such that $\mathbf{y}_{0}-\mathbf{x}_{0}$ is not proportional to $\mathbf{v}$. The function $\varphi(t)$ defined above now satisfies

$$
\varphi(0) \geqslant \varphi(1), \quad \varphi^{\prime}(0)=0, \quad \varphi^{\prime \prime}(0)=\left(\mathbf{y}_{0}-\mathbf{x}_{0}, D^{2} U\left(\mathbf{x}_{0}\right) \cdot\left(\mathbf{y}_{0}-\mathbf{x}_{0}\right)\right)>0 .
$$

Since $\varphi$ is not constant on $[0,1]$ (indeed, $\varphi^{\prime \prime}(0)>0$ ), the equality

$$
\varphi(1)-\varphi(0)=\int_{0}^{1} \varphi^{\prime}(s) \mathrm{d} s
$$

and the continuity of $\varphi^{\prime}$ implies that there is a point $t_{0} \in(0,1)$ such that $\varphi^{\prime}\left(t_{0}\right)<0$. By the mean value theorem, this in turn implies that there is a second point $t_{1} \in\left(0, t_{0}\right)$ such that

$$
0>\varphi^{\prime \prime}\left(t_{1}\right)=\left(\mathbf{y}_{0}-\mathbf{x}_{0}, D^{2} U\left(t_{1} \mathbf{y}_{0}+\left(1-t_{1}\right) \mathbf{x}_{0}\right) \cdot\left(\mathbf{y}_{0}-\mathbf{x}_{0}\right)\right) .
$$

This again contradicts the hypothesis, since $\mathbf{y}_{0}-\mathbf{x}_{0} \notin \mathbb{R} \mathbf{v}$.

\section{Appendix C. Evaluation of two sums involving the Weierstrass elliptic function}

In this appendix we shall evaluate the two sums

$$
S_{p}^{\prime}=\sum_{j=1}^{N-1} \wp_{N}^{p}(j), \quad p=1,2,
$$

which are used in Section 3 to compute the mean and the standard deviation of the spectrum of the Inozemtsev spin chain (2.8). In the latter equation $\wp_{N}$ denotes the Weierstrass function with periods $N$ and $2 \omega_{3}$, where $\omega_{3} \in \mathrm{i} \mathbb{R}_{+}$.

For the $p=1$ case, let $\gamma$ denote the (positively oriented) perimeter of the rectangle with vertices $\pm \omega_{3}$ and $N \pm \omega_{3}$, with a semicircular indentation of radius less than $\min \left(1,\left|\omega_{3}\right|\right)$ to the left of the points 0 and $N$ (see Fig. C.6. The function

$$
f(z)=\wp_{N}(z) \zeta_{1}(z),
$$

where $\zeta_{1}$ denotes the Weierstrass zeta function with periods 1 and $2 \omega_{3}$, is meromorphic in the complex plane with poles on the lattice $j+2 l \omega_{3}, j, l \in \mathbb{Z}$. The residues of $f$ at these points can be easily computed by taking into account Eq. A.6 and the well-known Laurent series

$$
\zeta_{1}(z)=\frac{1}{z}+\left[z^{3}\right]
$$

Indeed

$$
\wp_{N}(z) \zeta_{1}(z)=\frac{1}{z^{3}}+[z] \Longrightarrow \operatorname{Res}(f, 0)=0
$$


while at $z=j \in\{1, \ldots, N-1\}$ the analyticity of $\wp_{N}$ and the quasi-periodicity of $\zeta_{1}$ imply

$$
\wp_{N}(z) \zeta_{1}(z)=\wp_{N}(z)\left(\zeta_{1}(z-j)+2 j \zeta_{1}(1 / 2)\right) \Longrightarrow \operatorname{Res}(f, j)=\wp_{N}(j), \quad j=1, \ldots, N-1 .
$$

Applying Cauchy's residue theorem we obtain

$$
\frac{1}{2 \pi \mathrm{i}} \int_{\gamma} f(z) \mathrm{d} z=\sum_{j=1}^{N-1} \wp_{N}(j) \equiv S_{1}^{\prime} .
$$

The integral in the LHS can be readily computed taking into account the (quasi-)periodicity properties of the Weierstrass functions. Indeed, denoting by $L_{1}$ and $L_{2}$ the bottom and left sides of the contour $\gamma$, by Eqs. (A.2) we have

$$
\begin{aligned}
\int_{\gamma} f(z) \mathrm{d} z & =\int_{L_{1}} \wp_{N}(z)\left(\zeta_{1}(z)-\zeta_{1}\left(z+2 \omega_{3}\right)\right) \mathrm{d} z+\int_{L_{2}} \wp_{N}(z)\left(\zeta_{1}(z)-\zeta_{1}(z+N)\right) \mathrm{d} z \\
& =-2 \zeta_{1}\left(\omega_{3}\right) \int_{L_{1}} \wp_{N}(z) \mathrm{d} z-2 N \zeta_{1}(1 / 2) \int_{L_{2}} \wp_{N}(z) \mathrm{d} z \\
& =\left.2 \zeta_{1}\left(\omega_{3}\right) \zeta_{N}(z)\right|_{-\omega_{3}} ^{N-\omega_{3}}+\left.2 N \zeta_{1}(1 / 2) \zeta_{N}(z)\right|_{\omega_{3}} ^{-\omega_{3}}=4\left[\zeta_{1}\left(\omega_{3}\right) \zeta_{N}(N / 2)-N \zeta_{1}(1 / 2) \zeta_{N}\left(\omega_{3}\right)\right] .
\end{aligned}
$$

From Legendre's relation A.5 applied to both $\zeta_{1}$ and $\zeta_{N}$ we have

$$
\begin{aligned}
4 \omega_{3}\left[\zeta_{1}\left(\omega_{3}\right) \zeta_{N}(N / 2)-N \zeta_{1}(1 / 2) \zeta_{N}\left(\omega_{3}\right)\right] & =4 \zeta_{1}\left(\omega_{3}\right)\left(\frac{N}{2} \zeta_{N}\left(\omega_{3}\right)+\frac{\mathrm{i} \pi}{2}\right)-4 N \zeta_{N}\left(\omega_{3}\right)\left(\frac{1}{2} \zeta_{1}\left(\omega_{3}\right)+\frac{\mathrm{i} \pi}{2}\right) \\
& =2 \pi \mathrm{i}\left(\zeta_{1}\left(\omega_{3}\right)-N \zeta_{N}\left(\omega_{3}\right)\right) .
\end{aligned}
$$

Using this identity in Eq. C.3] we finally obtain the formula

$$
S_{1}^{\prime}=\frac{1}{\omega_{3}}\left(\zeta_{1}\left(\omega_{3}\right)-N \zeta_{N}\left(\omega_{3}\right)\right)
$$

With the help of Legendre's identity, we can rewrite this equation in the equivalent form

$$
S_{1}^{\prime}=2\left(\zeta_{1}(1 / 2)-\zeta_{N}(N / 2)\right) \text {. }
$$

In order to evaluate the sum (C.1) for $p=2$, consider to begin with the function

$$
g(z)=\sum_{j=0}^{N-1} \wp_{N}(z+j) .
$$

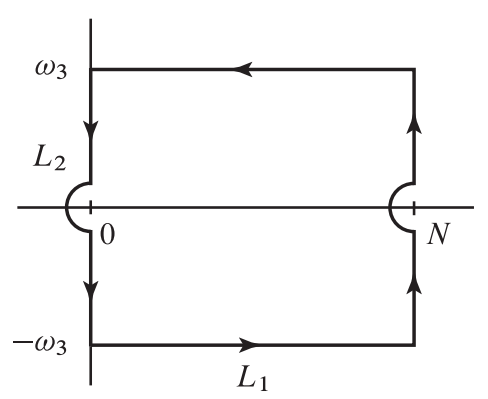

Figure C.6: Contour of integration used in the computation of the sum $S_{1}^{\prime}$. 
This function is easily seen to have periods 1 and $2 \omega_{3}$, and is analytic everywhere except for double poles at the points $j+2 l \omega_{3}(j, l \in \mathbb{Z})$ on its fundamental period lattice. Moreover, by Eq. A.6) the principal part of $g$ at these poles is $(z-j)^{-2}$. Since the Weierstrass function $\wp_{1}(z)$ with periods 1 and $2 \omega_{3}$ has the same poles and principal parts as $g$, by Liouville's theorem we have

$$
\sum_{j=0}^{N-1} \wp_{N}(z+j)=\wp_{1}(z)+c
$$

(where the constant $c$ is in fact the sum $S_{1}^{\prime}$ ). Differentiating this equality twice and taking into account the identity

$$
\wp^{\prime \prime}=6 \wp^{2}-\frac{1}{2} g_{2}
$$

we immediately obtain

$$
\sum_{j=1}^{N-1} \wp_{N}^{2}(z+j)=\wp_{1}^{2}(z)-\wp_{N}^{2}(z)+\frac{N}{12} g_{2}\left(N / 2, \omega_{3}\right)-\frac{1}{12} g_{2}\left(1 / 2, \omega_{3}\right) .
$$

The limit as $z \rightarrow 0$ of the LHS is the sum $S_{2}^{\prime}$. As to the RHS, using the Laurent series A.6 we readily find

$$
\lim _{z \rightarrow 0}\left[\wp_{1}^{2}(z)-\wp_{N}^{2}(z)\right]=\frac{1}{10}\left[g_{2}\left(1 / 2, \omega_{3}\right)-g_{2}\left(N / 2, \omega_{3}\right)\right] .
$$

From Eq. C.5 we thus obtain

$$
\sum_{j=1}^{N-1} \wp_{N}^{2}(j)=\frac{1}{12}\left(N-\frac{6}{5}\right) g_{2}\left(N / 2, \omega_{3}\right)+\frac{1}{60} g_{2}\left(1 / 2, \omega_{3}\right) .
$$

Proceeding in the same fashion, it is straightforward (albeit lengthy, unless $p$ is small) to compute the sum $S_{p}^{\prime}$ for any fixed value of $p$. For arbitrary $p$, it is not hard to show that

$$
S_{p}^{\prime}=\frac{(2 \pi)^{2 p}\left|B_{2 p}\right|}{(2 p) !}+O\left(N^{-1}\right),
$$

where $B_{n}$ denotes the $n$-th Bernoulli number. The proof is based on the absolute convergence in the punctured disk $0<|z|<\min (1, \tau)$ of the Laurent series [51]

$$
\wp(z ; 1 / 2, \mathrm{i} \tau / 2) \equiv \wp(z)=\frac{1}{z^{2}}+\sum_{l=1}^{\infty}(2 l+1) E_{2 l+2}(\tau) z^{2 l},
$$

where $E_{j}$ is the Eisenstein series

$$
E_{j}(\tau)=\sum_{(l, n) \in \mathbb{Z}^{2} \backslash\{0\}}(l+\mathrm{i} n \tau)^{-j}, \quad j>2 .
$$

Indeed, suppose first that $k^{2} \leqslant 1 / 2$, so that $\tau \equiv K^{\prime} / K \geqslant 1$. We then have

$\wp_{N}(j) \equiv \wp(j ; N / 2, \mathrm{i} N \tau / 2)=N^{-2} \wp(j / N)=\frac{1}{j^{2}}+\frac{1}{N^{2}} \sum_{l=1}^{\infty}(2 l+1) E_{2 l+2}(\tau)(j / N)^{2 l}, \quad 1 \leqslant j \leqslant N-1$, 
since in this case the series in the RHS of Eq. C.8 converges inside the unit disk. Moreover, from the absolute convergence of the latter series in its disk of convergence it follows that

$$
\left|\wp_{N}(j)-\frac{1}{j^{2}}\right| \leqslant \frac{1}{N^{2}} \sum_{l=1}^{\infty}(2 l+1)\left|E_{2 l+2}(\tau)\right|(1 / 2)^{2 l}=O\left(N^{-2}\right), \quad 1 \leqslant j \leqslant[N / 2],
$$

and therefore

$$
\wp_{N}^{p}(j)=\frac{1}{j^{2 p}}+O\left(N^{-2}\right), \quad 1 \leqslant j \leqslant[N / 2] .
$$

Summing over $j$ and taking into account that $\wp_{N}(j)=\wp_{N}(N-j)$ we easily obtain

$$
\begin{aligned}
S_{p}^{\prime} & =2 \sum_{j=1}^{[N / 2]} \wp_{N}^{p}(j)+(\pi(N)-1) \wp_{N}^{p}(N / 2)=2 \sum_{j=1}^{[N / 2]} \frac{1}{j^{2 p}}+O\left(N^{-1}\right)+(\pi(N)-1) N^{-2 p} \wp^{p}(1 / 2) \\
& =2 \zeta(2 p)+O\left(N^{-1}\right),
\end{aligned}
$$

where $\zeta$ denotes Riemann's zeta function ${ }^{8}$. This is essentially Eq. (C.77).

Suppose, on the other hand, that $k^{2}>1 / 2$, so that $\tau=K^{\prime} / K<1$. In this case we can write

$$
S_{p}^{\prime}=2 \sum_{j=1}^{[N \tau / 2]} \wp_{N}^{p}(j)+(\pi(N)-1) N^{-2 p} \wp^{p}(1 / 2)+R_{p},
$$

with

$$
R_{p}=2 \sum_{j=[N \tau / 2]+1}^{[N / 2]} \wp_{N}^{p}(j) \leqslant(N(1-\tau)+2) \wp_{N}^{p}(N \tau / 2)=(N(1-\tau)+2) N^{-2 p} \wp^{p}(\tau / 2)=O\left(N^{1-2 p}\right) .
$$

On the other hand, for $j=1, \ldots,[N \tau / 2]$ we again have

$$
\left|\wp_{N}(j)-\frac{1}{j^{2}}\right| \leqslant \frac{1}{N^{2}} \sum_{l=1}^{\infty}(2 l+1)\left|E_{2 l+2}(\tau)\right|(\tau / 2)^{2 l}=O\left(N^{-2}\right),
$$

on account of the absolute convergence of the power series in the RHS of Eq. (C.8) inside its disk of convergence $|z|<\tau$. Thus in this case

$$
\wp_{N}^{p}(j)=\frac{1}{j^{2 p}}+O\left(N^{-2}\right), \quad 1 \leqslant j \leqslant[N \tau / 2],
$$

from which Eq. C.7p easily follows as before.

\section{Appendix D. Simplification of several sums appearing in Eqs. 3.24 and 3.31}

In this appendix we shall simplify several sums appearing in Eqs. (3.24)-(3.31) for the skewness and kurtosis of the spectrum of a spin chain of the form (3.8). Consider, to begin, with, the last sum in Eq. 3.24, which can be written as

$$
\begin{aligned}
& \sum_{i, j, l}^{\prime} h_{i j} h_{j l} h_{l i}=6 \sum_{i<j<l} h(j-i) h(l-j) h(l-i)=6 \sum_{2 \leqslant a+b \leqslant N-1}(N-a-b) h(a) h(b) h(a+b) . \\
& { }^{8} \text { Recall that if } \lambda>0 \text { we have } \sum_{j=\lambda N+1}^{\infty} j^{-2 p}<\int_{\lambda N}^{\infty} x^{-2 p} \mathrm{~d} x=O\left(N^{1-2 p}\right) .
\end{aligned}
$$


Performing the change of index $a \mapsto c=N-a-b$ in the latter sum and making use of the identities (3.2) satisfied by the function $h$ we obtain

$$
\sum_{2 \leqslant a+b \leqslant N-1}(N-a-b) h(a) h(b) h(a+b)=\sum_{2 \leqslant b+c \leqslant N-1} c h(b) h(c) h(b+c),
$$

and therefore

$$
\sum_{2 \leqslant a+b \leqslant N-1} a h(a) h(b) h(a+b)=\frac{N}{3} \sum_{2 \leqslant a+b \leqslant N-1} h(a) h(b) h(a+b) .
$$

We thus have

$$
\sum_{i, j, l}^{\prime} h_{i j} h_{j l} h_{l i}=2 N \sum_{2 \leqslant a+b \leqslant N-1} h(a) h(b) h(a+b)=2 N \sum_{1 \leqslant i<j \leqslant N-1} h(i) h(j) h(j-i) .
$$

Consider next the second sum in Eq. 3.31):

$$
\begin{aligned}
\sum_{i, j, l}^{\prime} h_{i j}^{2} h_{j l}^{2} & =2 \sum_{i<j<l}\left[h^{2}(j-i) h^{2}(l-j)+h^{2}(j-i) h^{2}(l-i)+h^{2}(l-j) h^{2}(l-i)\right] \\
& =2 \sum_{2 \leqslant a+b \leqslant N-1}(N-a-b)\left[h^{2}(a) h^{2}(b)+h^{2}(a) h^{2}(a+b)+h^{2}(b) h^{2}(a+b)\right] \\
& =2 \sum_{2 \leqslant a+b \leqslant N-1} a\left[h^{2}(a) h^{2}(b)+h^{2}(a) h^{2}(a+b)+h^{2}(b) h^{2}(a+b)\right] .
\end{aligned}
$$

Hence

$$
\begin{aligned}
\sum_{2 \leqslant a+b \leqslant N-1}(N-a-b) & {\left[h^{2}(a) h^{2}(b)+h^{2}(a) h^{2}(a+b)+h^{2}(b) h^{2}(a+b)\right] } \\
& =\frac{N}{3} \sum_{\substack{2 \leqslant a+b \leqslant N-1\\
}}\left[h^{2}(a) h^{2}(b)+2 h^{2}(a) h^{2}(a+b)\right] \\
& =\frac{N}{6} \sum_{\substack{1 \leqslant a, b \leqslant N-1 \\
a+b \neq N}} h^{2}(a) h^{2}(b)+\frac{N}{3} \sum_{\substack{1 \leqslant a, b \leqslant N-1 \\
a \neq b}} h^{2}(a) h^{2}(b)=\frac{N}{2}\left(S_{2}^{2}-S_{4}\right),
\end{aligned}
$$

and therefore

$$
\sum_{i, j, l}^{\prime} h_{i j}^{2} h_{j l}^{2}=N\left(S_{2}^{2}-S_{4}\right) .
$$

The third sum in Eq. 3.31 can be dealt with similarly:

$$
\begin{aligned}
\sum_{i, j, l}^{\prime} h_{i j}^{2} h_{j l} h_{l i} & =2 \sum_{i<j<l}\left[h^{2}(j-i) h(l-j) h(l-i)+h^{2}(l-i) h(j-i) h(l-j)+h^{2}(l-j) h(j-i) h(l-i)\right] \\
& =2 \sum_{1 \leqslant a+b \leqslant N-1}(N-a-b)\left[h^{2}(a) h(b) h(a+b)+h^{2}(a+b) h(a) h(b)+h^{2}(b) h(a) h(a+b)\right] \\
& =\frac{2 N}{3} \sum_{1 \leqslant a+b \leqslant N-1}\left[h^{2}(a+b) h(a) h(b)+2 h^{2}(a) h(b) h(a+b)\right] \\
& =\frac{2 N}{3} \sum_{1 \leqslant i<j \leqslant N-1}\left[h^{2}(j) h(i) h(j-i)+2 h^{2}(i) h(j) h(j-i)\right] \\
& =2 N \sum_{1 \leqslant i<j \leqslant N-1} h^{2}(i) h(j) h(j-i)=2 N \sum_{1 \leqslant i<j \leqslant N-1} h^{2}(j) h(i) h(j-i),
\end{aligned}
$$


where in the last two steps we have made use of the identity $h(l)=h(N-l)$. Likewise, the last (cyclic) sum in Eq. 3.31) can be written as

$$
\begin{aligned}
\sum_{i, j, l, n}^{\prime} h_{i j} h_{j l} h_{l n} h_{n i}=8 \sum_{i<j<l<n}[h(j-i) h(l-j) h(n-l) h(n-i) \\
\quad+h(j-i) h(l-i) h(n-j) h(n-l)+h(l-i) h(l-j) h(n-i) h(n-j)],
\end{aligned}
$$

where each of the sums in the RHS can be easily simplified. Indeed, for the first of these sums we have

$$
\begin{aligned}
\sum_{i<j<l<n} h(j-i) h(l-j) h(n-l) h(n-i) & =\sum_{3 \leqslant a+b+c \leqslant N-1}(N-a-b-c) h(a) h(b) h(c) h(a+b+c) \\
& =\frac{N}{4} \sum_{3 \leqslant a+b+c \leqslant N-1} h(a) h(b) h(c) h(a+b+c),
\end{aligned}
$$

and similarly for the remaining two sums:

$$
\begin{aligned}
& \sum_{i<j<l<n}[h(j-i) h(l-i) h(n-j) h(n-l)+h(l-i) h(l-j) h(n-i) h(n-j)] \\
& =\sum_{3 \leqslant a+b+c \leqslant N-1}(N-a-b-c) h(a) h(a+c)[h(b) h(b+c)+h(a+b) h(a+b+c)] \\
& =\frac{N}{4} \sum_{3 \leqslant a+b+c \leqslant N-1} h(a) h(a+c)[h(b) h(b+c)+h(a+b) h(a+b+c)] \\
& =\frac{N}{2} \sum_{3 \leqslant a+b+c \leqslant N-1} h(a) h(b) h(a+c) h(b+c),
\end{aligned}
$$

where the last equality follows from the change of index $b \mapsto b^{\prime}=N-a-b-c$ in the second sum. We thus finally obtain

$$
\sum_{i, j, l, n}^{\prime} h_{i j} h_{j l} h_{l n} h_{n i}=2 N \sum_{3 \leqslant a+b+c \leqslant N-1} h(a) h(b)[h(c) h(a+b+c)+2 h(a+c) h(b+c)] .
$$

\section{References}

[1] F. D. M. Haldane, Phys. Rev. Lett. 60 (1988) 635-638.

[2] B. S. Shastry, Phys. Rev. Lett. 60 (1988) 639-642.

[3] M. Fowler, J. A. Minahan, Phys. Rev. Lett. 70 (1993) 2325-2328.

[4] F. D. M. Haldane, Z. N. C. Ha, J. C. Talstra, D. Bernard, V. Pasquier, Phys. Rev. Lett. 69 (1992) 2021-2025

[5] F. Finkel, A. González-López, Phys. Rev. B 72 (2005) 174411(6).

[6] B. Basu-Mallick, N. Bondyopadhaya, K. Hikami, SIGMA 6 (2010) 091(13).

[7] Z. N. C. Ha, F. D. M. Haldane, Phys. Rev. B 46 (1992) 9359-9368.

[8] A. P. Polychronakos, Phys. Rev. Lett. 70 (1993) 2329-2331.

[9] B. Sutherland, Phys. Rev. A 4 (1971) 2019-2021.

[10] B. Sutherland, Phys. Rev. A 5 (1972) 1372-1376.

[11] F. Calogero, J. Math. Phys. 12 (1971) 419-436.

[12] J. A. Minahan, A. P. Polychronakos, Phys. Lett. B 302 (1993) 265-270.

[13] V. I. Inozemtsev, D. V. Meshcheryakov, Phys. Scr. 33 (1986) 99-104.

[14] V. I. Inozemtsev, Phys. Scr. 53 (1996) 516-520.

[15] H. Frahm, J. Phys. A: Math. Gen. 26 (1993) L473-L479. 
[16] H. Frahm, V. I. Inozemtsev, J. Phys. A: Math. Gen. 27 (1994) L801-L807.

[17] M. A. Olshanetsky, A. M. Perelomov, Phys. Rep. 94 (1983) 313-404.

[18] J. Dittrich, V. I. Inozemtsev, J. Phys. A: Math. Gen. 26 (1993) L753-L756.

[19] G. Felder, A. Varchenko, Comp. Math. 107 (1997) 143-175.

[20] K. Takemura, Lett. Math. Phys. 53 (2000) 181-194.

[21] Y. Komori, K. Takemura, Commun. Math. Phys. 227 (2002) 93-118.

[22] E. Langmann, Lett. Math. Phys. 94 (2010) 63-75.

[23] J. Dittrich, V. I. Inozemtsev, Regul. Chaotic Dyn. 14 (2009) 218-222.

[24] J. C. Barba, V. I. Inozemtsev, Phys. Lett. A 372 (2008) 5951.

[25] V. I. Inozemtsev, J. Stat. Phys. 59 (1990) 1143-1155.

[26] D. Serban, M. Staudacher, JHEP 06 (2004) 001(31).

[27] T. Bargheer, N. Beisert, F. Loebbert, J. Phys. A: Math. Theor. 42 (2009) 285205(58).

[28] A. Rej, Lett. Math. Phys. 99 (2012) 85-102.

[29] D. Serban, JHEP 01 (2013) 012(16).

[30] V. I. Inozemtsev, Lett. Math. Phys. 36 (1996) 55-63.

[31] V. I. Inozemtsev, Phys. Part. Nucl. 34 (2003) 166-193.

[32] M. V. Berry, M. Tabor, Proc. R. Soc. London Ser. A 356 (1977) 375-394.

[33] O. Bohigas, M. J. Giannoni, C. Schmit, Phys. Rev. Lett. 52 (1984) 1-4.

[34] D. Poilblanc, T. Ziman, J. Bellissard, F. Mila, J. Montambaux, Europhys. Lett. 22 (1993) 537-542.

[35] A. Relaño, J. M. G. Gómez, R. A. Molina, J. Retamosa, E. Faleiro, Phys. Rev. Lett. 89 (2002) 244102(4).

[36] E. Faleiro, J. M. G. Gómez, R. A. Molina, L. Muñoz, A. Relaño, J. Retamosa, Phys. Rev. Lett. 93 (2004) 244101(4)

[37] D. Bernard, Int. J. Mod. Phys. B 7 (1993) 3517-3530.

[38] A. Enciso, F. Finkel, A. González-López, Phys. Rev. E 82 (2010) 051117(6).

[39] B. Berndt, B. P. Yeap, Adv. Appl. Math. 29 (2002) 358-385.

[40] A. P. Polychronakos, Nucl. Phys. B 419 (1994) 553-566.

[41] F. W. J. Olver, D. W. Lozier, R. F. Boisvert, C. W. Clark (Eds.), NIST Handbook of Mathematical Functions, Cambridge Univeristy Press, 2010.

[42] A. P. Polychronakos, J. Phys. A: Math. Gen. 39 (2006) 12793-12845.

[43] A. Enciso, F. Finkel, A. González-López, M. A. Rodríguez, Nucl. Phys. B 707 (2005) 553-576.

[44] B. Basu-Mallick, N. Bondyopadhaya, Nucl. Phys. B 757 (2006) 280-302.

[45] J. C. Barba, F. Finkel, A. González-López, M. A. Rodríguez, Phys. Rev. B 77 (2008) 214422(10).

[46] J. C. Barba, F. Finkel, A. González-López, M. A. Rodríguez, Nucl. Phys. B 806 (2009) 684-714.

[47] B. Basu-Mallick, F. Finkel, A. González-López, Nucl. Phys. B 812 (2009) 402-423.

[48] B. Basu-Mallick, N. Bondyopadhaya, Phys. Lett. A 373 (2009) 2831-2836.

[49] B. Basu-Mallick, F. Finkel, A. González-López, Nucl. Phys. B 866 (2013) 391-413.

[50] M. Abramowitz, I. A. Stegun, Handbook of Mathematical Functions, Dover, New York, ninth edition, 1970.

[51] E. M. Stein, R. Shakarchi, Complex Analysis, Princeton University Press, Princeton, N.J., 2003.

[52] F. Haake, Quantum Signatures of Chaos, Springer-Verlag, Berlin, second edition, 2001.

[53] M. L. Mehta, Random Matrices, Elsevier, San Diego, 3rd edition, 2004.

[54] J.-C. A. d'Auriac, J.-M. Maillard, C. M. Viallet, J. Phys. A: Math. Gen. 35 (2002) 4801-4822.

[55] J. C. Barba, F. Finkel, A. González-López, M. A. Rodríguez, Europhys. Lett. 83 (2008) 27005(6).

[56] M. S. Santhanam, J. N. Bandyopadhyay, Phys. Rev. Lett. 95 (2005) 114101(4).

[57] C. Male, G. Le Caër, R. Delannay, Phys. Rev. E 76 (2007) 042101(4).

[58] A. Relaño, Phys. Rev. Lett. 100 (2008) 224101(4).

[59] J. C. Barba, F. Finkel, A. González-López, M. A. Rodríguez, Nucl. Phys. B 839 (2010) 499-525.

[60] K. Hikami, Nucl. Phys. B 441 (1995) 530-548.

[61] F. Finkel, A. González-López, 2014. To be published.

[62] E. P. Miles, Jr, Am. Math. Mon. 67 (1960) 745-752.

[63] D. F. Lawden, Elliptic Functions and Applications, Springer-Verlag, Berlin, 1989. 